\title{
NUMERICAL COMPUTATIONS CONCERNING THE ERH
}

\author{
ROBERT RUMELY
}

In memory of D. H. Lehmer

\begin{abstract}
This paper describes a computation which established the ERH to height 10000 for all primitive Dirichlet $L$-series with conductor $Q \leq 13$, and to height 2500 for all $Q \leq 72$, all composite $Q \leq 112$, and other moduli. The computations were based on Euler-Maclaurin summation. Care was taken to obtain mathematically rigorous results: the zeros were first located within $10^{-12}$, then rigorously separated using an interval arithmetic package. A generalized Turing Criterion was used to show there were no zeros off the critical line. Statistics about the spacings between zeros were compiled to test the Pair Correlation Conjecture and GUE hypothesis.
\end{abstract}

\section{INTRODUCTION}

D. H. Lehmer was one of the pioneers in numerical computations concerning the Riemann zeta function: he showed [9], [10] that the first 25000 zeros of the zeta function were on the critical line. Since then, the Riemann Hypothesis has been checked to successively larger heights, the current record being $t=5 \cdot 10^{8}$ (van de Lune, te Riele, and Winter [11]); it has also been checked for long intervals at substantially greater heights by Odlyzko [16].

However, comparatively little energy has been spent on Dirichlet $L$-series and the Extended Riemann Hypothesis. This paper reports on a computation which established the ERH to height $t=10000$ for all primitive Dirichlet $L$-series with conductor $Q \leq 13$, and to height $t=2500$ for the following $Q$ :

- all $Q \leq 72$, all composite $Q \leq 112$;

- all $Q$ with $\varphi(Q) \leq 60$ ( $\varphi$ being Euler's function);

- all $Q$ of the form $M \cdot N$ with $M, N \leq 13$;

— all $Q$ dividing $432=2^{4} \cdot 3^{3}, 360=2^{3} \cdot 3^{2} \cdot 5,420=2^{2} \cdot 3 \cdot 5 \cdot 7$;

- prime powers $Q$ through $2^{8}, 3^{5}, 5^{3}, 7^{2}, 11^{2}, 13^{2}$;

- primes $Q$ for which $\mathbb{Q}(\sqrt{-Q})$ has class number 1 .

In addition to checking the ERH, the programs determined the zeros to within $10^{-12}$ and compiled statistics about them to test Montgomery's pair correlation conjecture and the GUE hypothesis for $L$-series. These moduli and heights were chosen to explore behavior depending on the conductor, order, and sign

Received by the editor July 24, 1991 and, in revised form, November 13, 1992.

1991 Mathematics Subject Classification. Primary 11M26, 11 M06.

Key words and phrases. Dirichlet L-series, Extended Riemann Hypothesis, GUE hypothesis, Pair correlation conjecture.

Work supported in part by National Science Foundation Grant DMS-8811507. 
of the characters; and also to (hopefully) be useful for applications requiring numerical approximations to the ERH.

The initial study of zeros of Dirichlet $L$-series was that of Davies and Haselgrove [3]. They computed the zeros to height $t=2000 / \varphi(Q)$ for real primitive characters with conductor $Q \in\{3,4,5,7,8,11,12,13,15,24\}$, and to various heights $t \leq 105$ for real and complex primitive characters with conductor $Q \in\{5,7,11,19,43,67,163\}$. Later Spira [24] computed the zeros to height $t=25$ for all real and complex primitive characters with $Q \leq 24$. More recently, Hejhal computed zeros at considerable heights for certain real primitive characters [5]. These investigations all supported the ERH.

The possibility for applications of such results has long been known: for example, McCurley [12], [13] used the Davies-Haselgrove zerofree region to prove a prime number theorem of Rosser-Schoenfeld type for the arithmetic progressions $x \equiv 1,2 \bmod (3)$. Recently, Ramaré [20], [21] extended McCurley's results to all the moduli $Q$ listed above, using the zerofree region described in this article. In [20] he applied this to reduce the bound on Schnirelman's constant from 19 to 13; very recently [22], he has further improved the bound to 7: that is, every integer $n \geq 2$ is a sum of at most 7 primes, and every even integer $n \geq 2$ is a sum of at most 6 primes.

In this investigation, great care was taken to obtain results which are mathematically rigorous, in so far as that can be done in a computer-assisted proof. The project had three phases. In phase I, the zeros on the line were located as accurately as possible. In phase II, the zeros from phase I were input to a program which recomputed function values at points between the zeros, using an interval arithmetic package to bound roundoff error, thus rigorously separating the zeros and providing a lower bound for the number of zeros on the line. In phase III, a generalized Turing criterion was used to show that there were no zeros off the line to a given height, and that all the zeros on the line had been located.

Let $\chi$ be a primitive Dirichlet character with conductor $Q$. Unless otherwise specified, we will assume throughout the paper that $Q>1$. Let $L(s, \chi)$ be the Dirichlet $L$-series attached to $\chi$; put

$$
\xi(s, \chi)=(Q / \pi)^{s / 2} \cdot \Gamma((s+\delta) / 2) \cdot L(s, \chi),
$$

where $\delta=(1-\chi(-1)) / 2$. Then (see, e.g., [7]) $\xi(s, \chi)$ is entire and satisfies the functional equation

$$
\xi(s, \chi)=W_{\chi} \cdot \xi(1-s, \bar{\chi})
$$

with $W_{\chi}=i^{-\delta} \tau(\chi) Q^{-1 / 2}$ and $\tau(\chi)=\sum_{a=1}^{Q} \chi(a) e^{a \cdot 2 \pi i / Q}$. The root number $W_{\chi}$ has absolute value 1 . If we write $s=\frac{1}{2}+i t$ and $W_{\chi}=e^{i \theta_{\chi}}$, it follows from the functional equation that if

$$
\theta(t, \chi)=(t / 2) \ln (Q / \pi)+\operatorname{Im}(\ln (\Gamma((s+\delta) / 2)))-\theta_{\chi} / 2
$$

then

$$
Z(t, \chi)=e^{i \theta(t, \chi)} \cdot L(s, \chi)
$$

is real-valued and has the same absolute value as $L(s, \chi)$. Hence, determining the zeros of $L(s, \chi)$ on the critical line reduces to a real-variable problem.

In $\S \S 1,2$, and 3 below we outline the three phases of the computation and describe the theory behind them. In $\S 4$ we examine the conclusions that can be 
drawn. In $\S 5$, which appears in the Supplement section at the end of this issue, we collect various tables and graphs.

The algorithms used by the program were based on Euler-Maclaurin summation. This was much simpler to implement than the Riemann-Siegel formula and made rigorous error bounds easy to obtain, though it limited the heights to which the computations could be carried.

The most interesting conclusion suggested by the data is that statistics about the zeros depend primarily on the set of primes dividing the conductor, and not on the conductor itself or the order or sign of the character. An example is given by Montgomery's pair correlation conjecture [15]. For a primitive $L(s, \chi)$ with conductor $Q$, Montgomery's conjecture predicts that (assuming the ERH), as $\frac{1}{2}+i \gamma_{n}$ runs over the zeros of $L(s, \chi)$ and $\gamma_{n} \rightarrow \infty$, the average number of zeros $\frac{1}{2}+i \gamma$ with $\gamma$ in the interval $\left(\gamma_{n}, \gamma_{n}+2 \pi \Delta / \ln (Q t / 2 \pi)\right)$ should be

$$
N(\Delta, \chi)=\int_{0}^{\Delta}\left(1-\frac{\sin ^{2}(\pi x)}{(\pi x)^{2}}\right) d x .
$$

We will call the integrand Montgomery's pair correlation function.

The data suggests that for each $Q$ there is an "empirical" pair correlation function (possibly depending on the height), which is the same for all primitive $L(s, \chi)$ with conductor $Q$, and is virtually the same for $Q$ with the same underlying prime divisors. In the range of $t$ studied, this function varies from modulus to modulus, and it agrees much better with Montgomery's function for prime moduli than for composite ones. For moduli divisible by 3, 4 and 12 the empirical pair correlation functions show large oscillations. Presumably they converge slowly to Montgomery's pair correlation function as $t$ increases, but further work would be needed to substantiate this. Odlyzko has proposed an explanation for these phenomena based on the Riemann-Siegel formula, which will be discussed in $\S 4$.

The computations were carried out on Zenith PC's in the Math 116 testing lab at the University of Georgia, and on the personal computers of several UGA faculty members. These machines were equipped with 8087 Math coprocessors, which were essential to the computation. In all, the computations represent more than a year's running time on 25 machines, with a combined speed of approximately 1 MFlop.

\section{COMPUting THE ZEROS}

Accurately locating the zeros of the $L(s, \chi)$ required many function evaluations. To compute the $L(s, \chi)$ efficiently, the program used polynomial approximations to the Taylor expansions

$$
L(s, \chi)=\sum_{n=0}^{\infty} a_{n}\left(s_{0}, \chi\right)\left(s-s_{0}\right)^{n}
$$

at points $s_{0}$ on the critical line. It proved convenient to move up the line by steps of $\frac{1}{2}$, taking $s_{0}=\frac{1}{2}, \frac{1}{2}+\frac{1}{2} i, \frac{1}{2}+i, \ldots$ and keeping the expansions at two successive points in memory at a given time. Enough terms of the Taylor series were kept that in the disc $D\left(s_{0}, \frac{1}{4}\right)$, the error due to the omitted terms was less than $10^{-20}$ (machine precision); in practice, this meant between 20 and 35 terms. 
A. Bounds for the number of Taylor coefficients. To bound the $a_{n}\left(s_{0}, \chi\right)$, and thus to determine the number of terms needed, one can use Cauchy's estimate

$$
\left|a_{n}\left(s_{0}, \chi\right)\right| \leq \frac{1}{2 \pi R^{n}} \int_{0}^{2 \pi}\left|L\left(s_{0}+\operatorname{Re}^{i \theta}, \chi\right)\right| d \theta .
$$

As $R$ varies, there is a tradeoff between the growth of $|L(s, \chi)|$ in the numerator and $R^{n}$ in the denominator; reasonable estimates were obtained by taking $R=3$ and using the following bounds for $|L(s, \chi)|$ on the strip $-2 \frac{1}{2} \leq$ $\operatorname{Re}(s) \leq 3 \frac{1}{2}:$

Lemma 1. If $\chi$ is a primitive Dirichlet character with conductor $Q>1$, then, writing $\sigma=\operatorname{Re}(s)$ one has:

$$
\begin{aligned}
& \text { for } 2 \frac{1}{2} \leq \sigma \leq 3 \frac{1}{2}, \quad|L(s, \chi)| \leq 1.3415 \\
& \text { for } 1 \frac{1}{2} \leq \sigma \leq 2 \frac{1}{2}, \quad|L(s, \chi)| \leq 2.6124 ; \\
& \text { for }-\frac{1}{2} \leq \sigma \leq 1 \frac{1}{2},|L(s, \chi)| \leq 2.6124 \cdot[Q /(2 \pi)]^{3 / 4-\sigma / 2} \cdot\left|s-2 \frac{1}{2}\right| \\
& \text { for }-1 \frac{1}{2} \leq \sigma \leq-\frac{1}{2},|L(s, \chi)| \leq 1.7416 \cdot[Q /(2 \pi)]^{1 / 2-\sigma} \cdot|s(s+2)| \\
& \text { for }-2 \frac{1}{2} \leq \sigma \leq-1 \frac{1}{2},|L(s, \chi)| \leq 1.1268 \cdot[Q /(2 \pi)]^{1 / 2-\sigma} \cdot|s(s+1)(s+3)|
\end{aligned}
$$

Proof. Trivially, $|L(s, \chi)| \leq \zeta(\sigma)$ for any $\sigma>1$, and computations show $\zeta\left(1 \frac{1}{2}\right) \leq 2.6124, \zeta\left(2 \frac{1}{2}\right) \leq 1.3415$. Since $\zeta(\sigma)$ is decreasing for $\sigma>1$, the first two assertions hold. Using the functional equations of $L(s, \chi)$ and $\Gamma(s)$, one finds that when $M$ is a positive integer,

$$
\left|L\left(\frac{1}{2}-M+i t, \chi\right)\right|=[Q /(2 \pi)]^{M} \cdot \prod_{k=0}^{M-1}\left|\frac{1}{2}+k+i t\right| \cdot\left|L\left(\frac{1}{2}+M+i t, \chi\right)\right| .
$$

To obtain the bound on the strip $-\frac{1}{2} \leq \sigma \leq 1 \frac{1}{2}$, apply the Phragmen-Lindelöf theorem to

$$
G(s)=\left(s-2 \frac{1}{2}\right)^{-1} \cdot[Q /(2 \pi)]^{s / 2-3 / 4} \cdot L(s, \chi) .
$$

By the formulas above, $|G(s)| \leq \zeta\left(1 \frac{1}{2}\right)$ on the lines $\sigma=-\frac{1}{2}, \sigma=1 \frac{1}{2}$. Trivially, $G(s)$ is holomorphic and has polynomial growth in the strip, so $|G(s)| \leq \zeta\left(1 \frac{1}{2}\right)$ throughout it. When $-1 \frac{1}{2} \leq \sigma \leq-\frac{1}{2}$, apply the Phragmen-Lindelöf theorem to

$$
G(s)=[s(s+2)]^{-1} \cdot[Q /(2 \pi)]^{s-1 / 2} \cdot L(s, \chi):
$$

on the line $\sigma=-1 \frac{1}{2},|G(s)| \leq \zeta\left(2 \frac{1}{2}\right) \leq 1.3415$; on the line $\sigma=-\frac{1}{2},|G(s)| \leq$ $\frac{2}{3} \cdot \zeta\left(1 \frac{1}{2}\right) \leq 1.7416$. When $-2 \frac{1}{2} \leq \sigma \leq-1 \frac{1}{2}$, apply Phragmen-Lindelöf to

$$
G(s)=[s(s+1)(s+3)]^{-1} \cdot[Q /(2 \pi)]^{s-1 / 2} \cdot L(s, \chi):
$$

on the line $\sigma=-2 \frac{1}{2},|G(s)| \leq \zeta\left(3 \frac{1}{2}\right) \leq 1.1268$; on the line $\sigma=-1 \frac{1}{2},|G(s)| \leq$ $\frac{2}{3} \cdot \zeta\left(2 \frac{1}{2}\right) \leq .8944$.

Lemma 2. Writing $s_{0}=\frac{1}{2}+i t$, put $\lambda=[Q /(2 \pi)] \cdot[|t|+4]$. Then $\left|a_{n}\left(s_{0}, \chi\right)\right| \leq 3^{-n} \cdot P(\lambda), \quad$ where $P(\lambda)=0.303 \lambda^{3}+0.221 \lambda^{2}+0.605 \lambda+0.687$.

Proof. Applying the Cauchy estimate, it is enough to show that

$$
(2 \pi)^{-1} \int_{0}^{2 \pi}\left|L\left(s_{0}+3 e^{i \theta}, \chi\right)\right| d \theta
$$


is bounded by $P(\lambda)$. We use the estimates for $|L(s, \chi)|$ in Lemma 1. One easily sees that on the arcs of $\partial D\left(s_{0}, 3\right)$ in $-2 \frac{1}{2} \leq \sigma \leq-1 \frac{1}{2},|s(s+1)(s+3)| \leq$ $(|t|+4)^{3}$; on the arcs in $-1 \frac{1}{2} \leq \sigma \leq-\frac{1}{2},|s(s+2)| \leq(|t|+4)^{2}$; and on the arcs in $-\frac{1}{2} \leq \sigma \leq 1 \frac{1}{2},\left|s-2 \frac{1}{2}\right| \leq 1.0607 \cdot(|t|+4)$. Using these bounds, together with the lengths of the arcs involved, one finds the stated formula.

This immediately yields

Proposition 1. Suppose $\operatorname{Re}\left(s_{0}\right)=\frac{1}{2}$. In order for $\sum_{n=0}^{N} a_{n}\left(s_{0}, \chi\right)\left(s-s_{0}\right)^{n}$ to approximate $L(s, \chi)$ within $10^{-20}$ on $D\left(s_{0}, \frac{1}{4}\right)$, it suffices that $N$ be large enough that $P(\lambda) /\left(11 \cdot 12^{N}\right) \leq 10^{-20}$.

Thus, if $Q=13$ and $t=10000$, then $N=31$ will do; if $Q=432$ and $t=2500$, then $N=33$ suffices. The programs determined the number of coefficients needed as it ran.

B. Computing the Taylor coefficients. To compute the $a_{n}\left(s_{0}, \chi\right)$, the program used the decomposition

$$
L(s, \chi)=\sum_{\substack{(a, Q)=1 \\ 1 \leq a<Q}} \chi(a) \cdot \zeta(s ; a, Q),
$$

where for $\operatorname{Re}(s)>1$

$$
\zeta(s ; a, Q)=\sum_{m=0}^{\infty} \frac{1}{(a+m Q)^{s}} .
$$

Thus,

$$
a_{n}\left(s_{0}, \chi\right)=(1 / n !) \cdot \sum_{a} \chi(a) \cdot(d / d s)^{n} \zeta\left(s_{0} ; a, Q\right) .
$$

Each partial zeta function $\zeta(s ; a, Q)$ has an analytic continuation to $\mathbb{C} \backslash\{1\}$ with a simple pole of residue $1 / Q$ at $s=1$. When $\operatorname{Re}(s)>1$,

$$
(-1)^{n} \cdot(d / d s)^{n} \zeta(s ; a, Q)=\sum_{m=0}^{\infty} \frac{\ln (a+m Q)^{n}}{(a+m Q)^{s}} .
$$

To evaluate $(d / d s)^{n} \zeta(s ; a, Q)$ for $\operatorname{Re}(s) \leq 1$, the program used EulerMaclaurin summation (see $[4, \S 6.2)]$ ); if we write

$$
\begin{aligned}
f_{n}(s ; x, a, Q) & =\frac{\ln (a+x Q)^{n}}{(a+x Q)^{s}}, \\
f_{n}^{(k)}(s ; x, a, Q) & =(d / d x)^{k}\left(\frac{\ln (a+x Q)^{n}}{(a+x Q)^{s}}\right),
\end{aligned}
$$

then for $\operatorname{Re}(s)>1-2 L$

$$
\begin{aligned}
(-1)^{n} \cdot(d / d s)^{n} \zeta(s ; a, Q) & \\
\approx & \sum_{m=1}^{M-1} f_{n}(s ; m, a, Q)+\frac{1}{2} f_{n}(s ; M, a, Q)+I_{n}(s ; M, a, Q) \\
& \quad-\sum_{\nu=1}^{L}\left(B_{2 \nu} /(2 \nu) !\right) \cdot f_{n}^{(2 \nu-1)}(s ; M, a, Q) .
\end{aligned}
$$


Here, $I_{n}(s ; M, a, Q)$ is the analytic continuation of the integral

$$
\int_{M}^{\infty} f_{n}(s ; x, a, Q) d x
$$

which converges for $\operatorname{Re}(s)>1$, and the $B_{2 \nu}$ are Bernoulli numbers $\left(B_{2}=1 / 6\right.$, $\left.B_{4}=-1 / 30, B_{6}=1 / 42, \ldots\right)$. The error in this approximation is

(2) $\quad R_{2 L}=R_{2 L}(s ; n, M, a, Q)=\frac{-1}{(2 \nu) !} \int_{M}^{\infty} \bar{B}_{2 L}(x) f_{n}^{(2 L)}(s ; x, a, Q) d x$,

where $\bar{B}_{2 L}(x)$ is the periodified Bernoulli polynomial (see [4, p. 101]) satisfying $\left|\bar{B}_{2 L}(x)\right| \leq\left|B_{2 L}\right|$ for all $x$. We will see below that $R_{2 L}$ can be made negligibly small if $M$ and $L$ are sufficiently large.

In formula (1), $I_{n}(s ; M, a, Q)$ can be evaluated using a recurrence:

$$
\begin{aligned}
I_{0}(s ; M, a, Q)= & \frac{1}{Q(s-1)(a+M Q)^{s-1}}, \\
I_{n}(s ; M, a, Q)= & {[\ln (a+M Q)]^{n} \cdot I_{0}(s ; M, a, Q) } \\
& +[n /(s-1)] \cdot I_{n-1}(s ; M, a, Q) .
\end{aligned}
$$

Likewise, the derivatives $f_{n}^{(k)}(s ; x, a, Q)$ can be evaluated using the recurrence

$$
\begin{aligned}
f_{n}^{(k)}(s ; x, a, Q)= & n \cdot Q \cdot f_{n-1}^{(k-1)}(s+1 ; x, a, Q) \\
& -s \cdot Q \cdot f_{n}^{(k-1)}(s+1 ; x, a, Q) .
\end{aligned}
$$

To estimate $R_{2 L}$, first note that for any $n$, if $\operatorname{Re}(s)=\sigma$, then $\left|f_{n}^{(0)}(s ; x, a, Q)\right|$ $=f_{n}^{(0)}(\sigma ; x, a, Q)$. Also, if $n_{1}<n_{2}$ and $a+x Q>1$, then $\left|f_{n_{1}}^{(0)}(s ; x, a, Q)\right|<$ $\left|f_{n_{2}}^{(0)}(s ; x, a, Q)\right|$. Using the recurrence above, one finds by induction that for $\sigma>0$

$$
\begin{aligned}
& \left|f_{n}^{(k)}(s ; x, a, Q)\right| \\
& \quad \leq(|s|+n)(|s+1|+n) \cdots(|s+k-1|+n) \cdot Q^{k} \cdot f_{n}^{(0)}(\sigma+k ; x, a, Q) .
\end{aligned}
$$

Furthermore, for any $\tau>1$, writing $B=a+M Q$, we get

$$
\int_{M}^{\infty} f_{n}^{(0)}(\tau ; x, a, Q) d x=\frac{[\ln (B)]^{n}}{Q(\tau-1) B^{\tau-1}} \sum_{j=0}^{n}(-1)^{j} \frac{n(n-1) \cdots(n-j+1)}{[(\tau-1) \ln (B)]^{j}} .
$$

If $|\tau-1| \cdot \ln (B)>2 n$, then the sum on the right has absolute value at most 2 . Inserting these estimates in the expression for $R_{2 L}$, one finds

$$
\begin{aligned}
\mid R_{2 L} & (s ; n, M, a, Q) \mid \\
& \leq\left|B_{2 L}\right| /(2 L) ! \int_{M}^{\infty}\left|f_{n}^{(2 L)}(s ; x, a, Q)\right| d x \\
& \leq(|s|+n) \cdots(|s+2 L-1|+n) \cdot Q^{2 L} \cdot \frac{\left|B_{2 L}\right|}{(2 L) !} \cdot \int_{M}^{\infty} f_{n}^{(0)}(\sigma+2 L ; x, a, Q) d x \\
& \leq \frac{2[\ln (a+M Q)]^{n}(M+a / Q)^{1-\sigma}}{Q^{\sigma}(\sigma+2 L-1)} \cdot \frac{\left|B_{2 L}\right|}{(2 L) !} \cdot \frac{(|s|+n) \cdots(|s+2 L-1|+n)}{(M+a / Q)^{2 L}},
\end{aligned}
$$

provided $(\sigma+2 L-1) \cdot \ln (a+M Q) \geq 2 n$. This side condition is easily met in practice. The program used $\sigma=\frac{1}{2}$ and $M \approx 1.3(|s|+40)$; since in any 
case $Q \geq 3$, it was enough to have $L \geq 9$ to satisfy the side condition for all $n \leq 35$.

The discussion above leads immediately to

Proposition 2. Suppose $\operatorname{Re}\left(s_{0}\right)=\frac{1}{2}$. To compute $(d / d s)^{n} \zeta\left(s_{0} ; a, Q\right)$ accurately enough that for $s \in D\left(s_{0}, \frac{1}{4}\right)$ each term $a_{n}\left(s_{0}, \chi\right) \cdot\left(s-s_{0}\right)^{n}$ contributes an error at most $10^{-20}$ to the sum $\sum_{n=0}^{N} a_{n}\left(s_{0}, \chi\right)\left(s-s_{0}\right)^{n}$, it is enough to choose $M$ and $L$ so that $\left(2 L-\frac{1}{2}\right) \cdot \ln (a+M Q) \geq 2 N$, and so that for $0 \leq n \leq N$,

$$
\begin{aligned}
& \frac{1}{\left(2 L-\frac{1}{2}\right)} \cdot \frac{\left|B_{2 L}\right|}{(2 L) !} \cdot \frac{(|s|+n) \cdots(|s+2 L-1|+n)}{(M+a / Q)^{2 L}} \\
& \quad \leq \frac{10^{-20} \cdot Q^{1 / 2} \cdot 4^{n} \cdot n !}{2 \cdot \varphi(Q) \cdot[\ln (a+M Q)]^{n} \cdot(M+a / Q)^{1 / 2}} .
\end{aligned}
$$

As noted, the program took $M \approx 1.3(|s|+40)$. Since $\left|B_{2 L}\right| /(2 L) ! \approx 2 /(2 \pi)^{2 L}$, as soon as $|s|$ is even moderately large, the left side in the estimate above is at most $1 /(8.16)^{2 L}$. Thus the condition is easy to satisfy. For example, if $N=32, Q=100,|s|=2500$, then $L=12$ will suffice.

C. Generating the characters. To generate the primitive characters with conductor $Q$, the program used the following procedure. Let the factorization of $Q$ into prime powers be $Q=p_{1}^{n_{1}} \cdots p_{r}^{n_{r}}$. Via the Chinese remainder theorem, there is a canonical isomorphism

$$
(\mathbb{Z} / Q \mathbb{Z})^{\times} \cong \prod_{i=1}^{r}\left(\mathbb{Z} / p_{i}^{n_{i}} \mathbb{Z}\right)^{\times} .
$$

Furthermore, for each $p_{i}>2$, there is a canonical isomorphism

$$
\begin{aligned}
\left(\mathbb{Z} / p_{i}^{n_{i}} \mathbb{Z}\right)^{\times} & \cong\left(\mathbb{Z} / p_{i} \mathbb{Z}\right)^{\times} \times\left(1+p_{i} \mathbb{Z}\right) /\left(1+p_{i}^{n_{i}} \mathbb{Z}\right), \\
\alpha\left(\bmod p_{i}^{n_{i}}\right) & \mapsto\left(\alpha\left(\bmod p_{i}\right), \operatorname{Teich}_{p_{i}}(\alpha)^{-1} \cdot \alpha\left(\bmod p_{i}^{n_{i}}\right)\right),
\end{aligned}
$$

where

$$
\operatorname{Teich}_{p_{i}}(\alpha)=\alpha^{p_{i}^{n_{i}}} \quad\left(\bmod p_{i}^{n_{i}}\right)
$$

is the "Teichmüller representative" of $\alpha\left(\bmod p_{i}^{n_{i}}\right)$. When $p_{i}=2$, there are no primitive characters $(\bmod Q)$ unless $n_{i} \geq 2$, and in that case there is a corresponding isomorphism

$$
\left(\mathbb{Z} / 2^{n_{i}} \mathbb{Z}\right)^{\times} \cong\{ \pm 1\} \times(1+4 \mathbb{Z}) /\left(1+2^{n_{i}} \mathbb{Z}\right) .
$$

Further, the $p$-adic logarithm maps, given by $\log _{p}(1+z)=\sum_{n=1}^{\infty}(-1)^{n+1} z^{n} / n$ for $z \in p \mathbb{Z}_{p}$ (resp. $z \in 4 \mathbb{Z}_{2}$, if $p=2$ ), induce canonical isomorphisms

$$
\begin{aligned}
\left(1+p_{i} \mathbb{Z}\right) /\left(1+p_{i}^{n_{i}} \mathbb{Z}\right) \cong\left(\mathbb{Z} / p_{i}^{n_{i}-1} \mathbb{Z}\right) & \left(\text { if } p_{i}>2, n_{i}>1\right), \\
(1+4 \mathbb{Z}) /\left(1+2^{n_{i}} \mathbb{Z}\right) \cong\left(\mathbb{Z} / 2^{n_{i}-2} \mathbb{Z}\right) & \left(\text { if } p_{i}=2, n_{i}>2\right) .
\end{aligned}
$$

For $p_{i}>2$, the groups $\left(\mathbb{Z} / p_{i} \mathbb{Z}\right)^{\times}$are cyclic, and though they do not have "arithmetically canonical" generators, each has a "computationally canonical" generator, namely the least positive primitive root $g_{i}$. The discrete logarithm, relative to $g_{i}$, gives an isomorphism $\left(\mathbb{Z} / p_{i} \mathbb{Z}\right)^{\times} \cong \mathbb{Z} /\left(p_{i}-1\right) \mathbb{Z}$. When $p_{i}=2$, then $(\mathbb{Z} / 4 \mathbb{Z})^{\times}=\{ \pm 1\} \cong \mathbb{Z} / 2 \mathbb{Z}$. 
By means of these isomorphisms, each $a \in(\mathbb{Z} / Q \mathbb{Z})^{\times}$encodes a vector $\vec{\Lambda}(a)$ in an appropriate product of the additive groups $\mathbb{Z} /\left(p_{i}-1\right) \mathbb{Z}, \mathbb{Z} / p_{i}^{n_{i}-1} \mathbb{Z}, \mathbb{Z} / 2 \mathbb{Z}$, $\mathbb{Z} / 2^{n_{i}-2} \mathbb{Z}$. For notational convenience, write this group as $\prod_{j=1}^{R}\left(\mathbb{Z} / m_{j} \mathbb{Z}\right)$, and let the $j$ th coordinate of $\vec{\Lambda}(a)$ be $\Lambda_{j}(a)$. We identify elements of $\mathbb{Z} / m_{j} \mathbb{Z}$ with their representatives in $\mathbb{Z}$ in the range $0 \leq x<m_{j}$.

Each $b \in(\mathbb{Z} / Q \mathbb{Z})^{\times}$defines a character $\chi_{b}:(\mathbb{Z} / Q \mathbb{Z})^{\times} \rightarrow \mathbb{C}^{\times}$via

$$
\chi_{b}(a)=\exp \left(2 \pi i \sum_{j=1}^{R} \Lambda_{j}(a) \cdot \Lambda_{j}(b) / m_{j}\right) .
$$

The various characters $\chi_{b}$ are distinct. For a given $b$, the character $\chi_{b}$ is primitive if and only if for each prime $p_{i}$ dividing $Q$ :

if $p_{i} \| Q$ (resp. $4 \| Q$ if $p_{i}=2$ ), then for the index $j$ such that $\mathbb{Z} / m_{j} \mathbb{Z}$ corresponds to $\left(\mathbb{Z} / p_{i} \mathbb{Z}\right)^{\times}\left(\right.$resp. $\{ \pm 1\}$ if $\left.p_{i}=2\right)$, $\Lambda_{j}(b) \neq 0$;

if $p_{i}^{n_{i}} \| Q$ with $n_{i}>1$ (resp. $n_{i}>2$ if $p_{i}=2$ ), then for the index $j$ such that $\mathbb{Z} / m_{j} \mathbb{Z}$ corresponds to $\left(1+p_{i} \mathbb{Z}\right) /\left(1+p_{i}^{n_{i}} \mathbb{Z}\right)$ (resp. $(1+4 \mathbb{Z}) /\left(1+2^{n_{i}} \mathbb{Z}\right)$ if $\left.p_{i}=2\right), \Lambda_{j}(b)$ is coprime to $p_{i}$.

Let $\lambda(Q)$ denote the number of primitive characters $(\bmod Q)$. By listing, in increasing order, the numbers $b_{1}, \ldots, b_{\lambda(Q)}\left(1 \leq b_{K} \leq Q\right)$ which encode primitive characters, the program assigned an "identification number" $K$ to each primitive character in such a way that $K \leftrightarrow \chi_{b_{K}}$. These numbers $K$ were used to identify the $L$-series in all output from the program.

It should be noted that this numbering scheme differs from that used by Davies-Haselgrove [3] and Spira [24]. The correspondence between the two numberings can easily be found on a case-by-case basis, but is not given by a simple formula.

D. Search method. Input to the program was the modulus $Q$ and (optionally) the initial height $t$. In a preprocessing phase, the program generated the primitive characters $\chi$ with conductor $Q$ and calculated the root number $W_{\chi}$ and other data about the $L$-series it needed. It kept a "restart file" so that if the search for zeros was interrupted, it could begin again where it left off. If no restart file was present, and no height was specified, the search began at $t=-15.0$.

In searching for the zeros, the program computed Taylor series at points $s_{0}=\frac{1}{2}+i t$ separated vertically by steps of size $1 / 2$. At a given $s_{0}$, it first computed the Taylor coefficients of the $\zeta(s ; a, Q)$ for all $a, 1 \leq a<Q$, with $(a, Q)=1$; it then computed the Taylor coefficients of the $L(s, \chi)$ for primitive characters $\chi$. The $L$-series coefficients at two successive points were kept in memory at a given time. Examining each $L(s, \chi)$ in turn, the program searched for zeros of the real-valued function $Z(t, \chi)=e^{i \theta(t, \chi)} L(s, \chi)$, using the Van Wijngaarden-Dekker-Brent rootfinding algorithm [18, p. 251], and searched for maxima and minima of $Z(t, \chi)$, using Brent's golden ratio/parabolic interpolation algorithm $[18$, p. 285]. The step size for these searches was about

$$
\frac{1}{15} \cdot \frac{2 \pi}{\ln (Q t /(2 \pi))} \text {. }
$$


The program used Stirling's formula for $\ln (\Gamma(z))$ to compute the quantities $\theta(t, \chi)$ to within $10^{-20}$. It was prepared to detect zeros off the line, and if it found successive maxima or minima of $Z(t, \chi)$ with the same sign, it used Laguerre's method [18, p. 265] to search for the nearest root of $L(s, \chi)$. (In practice, this routine was frequently invoked near $t=0$ and located the smallest trivial zero.)

While running, the program spent almost all $(98 \%+)$ of its time computing Taylor coefficients. At each $s_{0}$, after the coefficients had been calculated, it opened a file to record the zeros and maxima/minima that were found; when the searches had been completed for all $L(s, \chi)$, it closed that file and rewrote the "restart" file, recording the search height reached for each $L$-series. Frequently, different regions for the same modulus were searched by different machines, and at the end, the resulting zeros $/ \mathrm{max} / \mathrm{min}$ files were merged together.

\section{VALIDATION OF THE ZEROS}

Though the computations carried out in Phase I were (presumably) very accurate, they were not mathematically rigorous because no attempt was made to bound roundoff error. In order to rigorously prove the zeros were on the line, a second program was used, which took as its input the list of zeros from the first. It ordered them by increasing ordinates, then chose validation points (" $V$ points") intermediate to the zeros and well separated from them, at which to re-evaluate the $Z(t, \chi)$. By means of Euler-Maclaurin summation, it computed the $\zeta(s ; a, Q)$ and $L(s, \chi)$ directly (not their Taylor expansions), and then the $Z(t, \chi)$. This program took roughly $20 \%$ as long to run as the program in Phase I. Its code was written using an interval arithmetic package to bound the accumulated roundoff error, and hence to show that $Z(t, \chi)$ truly changed sign between each pair of $V$-points.

To understand this computation, it is first necessary to recall the specifications of the 8087 Math Chip. In extended precision mode, the chip computes using an 80-bit word, with a 1-bit sign, a 15-bit exponent, and a 64-bit mantissa. It can carry out the basic arithmetic operations and compute the basic exponential and trigonometric functions to 64-bit accuracy, with the result rounded nearest, up (towards $+\infty$ ), down (towards $-\infty$ ), or truncated towards 0 , as the user specifies. Intel states [6, p. 6-17]:

"Internally, the 8087 employs three extra bits (guard, round, and sticky bits) which enable it to represent the infinitely precise true result of a computation; these bits are not accessible to programmers. Whenever the destination can represent the infinitely precise true result, the 8087 delivers it. Rounding occurs in arithmetic and store operations when the format of the destination cannot exactly represent the infinitely precise true result. ... Given a true result $b$ that cannot be represented by the target data type, the 8087 determines the two representable numbers $a$ and $c$ that most closely bracket $b$ in value $(a<b<c)$. The processor then rounds [as specified in the processor control word] ... Rounding introduces an error in a result that is less than one unit in the last place to which the result is rounded." 
Assuming the 8087 performs correctly, it is possible to compute the $Z(t, \chi)$, giving a mathematically rigorous bound on the roundoff error, and hence to show that it truly changes sign on a given interval.

The author's interval arithmetic package used a representation of numbers consisting of a pair $\left(a, \varepsilon_{a}\right)$, where $a$ is the "most likely" or "main" value of a real number $\alpha$, and $\varepsilon_{a}$ is an "uncertainty radius": $\alpha$ is guaranteed to lie in the interval $\left(a-\varepsilon_{a}, a+\varepsilon_{a}\right)$. Using calculus, it is easy to determine how uncertainties propagate through basic operations and functions. For example, for addition, $\left(a, \varepsilon_{a}\right)+\left(b, \varepsilon_{b}\right)=\left(c, \varepsilon_{c}\right)$, with $c=a+b$ (rounded nearest), and $\varepsilon_{c}=\varepsilon_{a}+\varepsilon_{b}+\varepsilon_{r o}$ (rounded up), where $\varepsilon_{r o}$ is the roundoff error in $c$, taken to be the least significant bit of $c$. In considering these formulas, it is important to remember that in the chip's internal representation, $a$ and $b$ are definite numbers and that the information needed to give $a+b$ to 64-bit accuracy is available. The formulas used in the package are given in Table 2.1.

Granted the basic operations for real numbers with uncertainties, the program represented complex numbers as ordered pairs of such numbers, and then computed the necessary character, partial zeta function, $\Gamma$-function, and $L$ series values, keeping track of roundoff error and any truncation errors in the formulas for the functions.

Over the entire calculation, the smallest value of $|Z(t, \chi)|$ found at any $V$ point was approximately $4.2 \times 10^{-6}$ and the largest uncertainty radius was about $2.2 \times 10^{-11}$. Assuming the computations are correct, they give a mathematical proof that there are at least as many zeros on the line as claimed. It is also possible to analyze the code, using the methods of backwards error analysis, and to establish a theoretical bound for the roundoff error in $Z(t, \chi)$ as given by the main value computation:

$$
\left[11 Q^{2} t^{3 / 2}+194 Q^{1 / 2} t^{2}+206 Q t^{3 / 2}+23 Q^{3 / 2} t+11536 Q^{1 / 2} t^{1 / 2} \ln (t)\right] \cdot u
$$

where $u=2^{-63}$ is the basic "unit of accuracy" of the computer. For all heights and moduli considered here, the bound is less than $4.3 \times 10^{-8}$. This provides an alternative proof for the count of zeros on the line, with the advantage that it only relies on the accuracy of the main value computations, and not the uncertainty intervals.

It is of course a question whether the computations really do provide a proof. They might be criticized from at least four directions: the correctness of the algorithms used, the correctness of their implementation, the correctness of the 8087's internal design, and the correct functioning of the machines. The interval arithmetic package was subjected to a test program which repeatedly checked several hundred identities on random inputs of all sizes (identities like $(a+b)^{2}=$ $a^{2}+2 a b+b^{2},(a * b) / b=a, \operatorname{sqrt}(x)=\exp \left(\frac{1}{2} \ln (x)\right), \sin ^{2}(x)+\cos ^{2}(x)=1$, etc. $)$ During a several-hour run, no violations of the uncertainty bounds were found. The output of the programs for computing $L$-series was subject to both internal and external checks. Internally, as $Z(t, \chi)=e^{i \theta(t, \chi)} L(s, \chi)$ is provably realvalued but was computed using complex arithmetic, its imaginary part could be compared with 0 . The results were consistent with expected roundoff errors, typically being near $10^{-18}$ for small $t$, and gradually increasing to $10^{-14}$ for $t \approx 2500$. Externally, the lists of zeros produced were checked against earlier published lists [3, 4, 24] and found to be consistent. The 8087 chip has a proprietary design which cannot be examined; however it has been given IEEE 
TABLE 2.1. Formulas used in the Interval Arithmetic package

\begin{tabular}{|c|c|}
\hline Operation $^{1), 3)}$ & Uncertainty radius $^{1), 2), 3)}$ \\
\hline$\left(c, \varepsilon_{c}\right)=\left(a, \varepsilon_{a}\right)+\left(b, \varepsilon_{b}\right)$ & $\varepsilon_{c}=\varepsilon_{a}+\varepsilon_{b}+\varepsilon_{\mathrm{ro}}$ \\
\hline$\left(c, \varepsilon_{c}\right)=\left(a, \varepsilon_{a}\right)-\left(b, \varepsilon_{b}\right)$ & $\varepsilon_{c}=\varepsilon_{a}+\varepsilon_{b}+\varepsilon_{\mathrm{ro}}$ \\
\hline$\left(c, \varepsilon_{c}\right)=\left(a, \varepsilon_{a}\right) \cdot\left(b, \varepsilon_{b}\right)$ & $\varepsilon_{c}=|a| \cdot \varepsilon_{b}+|b| \cdot \varepsilon_{a}+\varepsilon_{a} \cdot \varepsilon_{b}+\varepsilon_{\mathrm{ro}}$ \\
\hline$\left(c, \varepsilon_{c}\right)=\left(a, \varepsilon_{a}\right) /\left(b, \varepsilon_{b}\right)$ & $\varepsilon_{c}=|a| \cdot \varepsilon_{b} /\left[|b| \cdot\left(|b|-\varepsilon_{b}\right)\right]$ \\
\hline$\left(\right.$ where $\left.|b|>e_{b}\right)$ & $+\varepsilon_{a} /\left(|b|-\varepsilon_{b}\right)+\varepsilon_{\mathrm{ro}}$ \\
\hline $\begin{aligned}\left(c, \varepsilon_{c}\right)= & \operatorname{sqrt}\left(a, \varepsilon_{a}\right) \\
& \left(\text { where } a \geq \varepsilon_{a}\right)\end{aligned}$ & $\varepsilon_{c}=\operatorname{sqrt}(a)_{\mathrm{up}}-\operatorname{sqrt}\left(a-\varepsilon_{a}\right)_{\mathrm{down}}+\varepsilon_{\mathrm{ro}}$ \\
\hline$\left(c, \varepsilon_{c}\right)=\ln \left(a, \varepsilon_{a}\right)$ & $\varepsilon_{c}=\varepsilon_{a} /\left(a-\varepsilon_{a}\right)+\varepsilon_{\mathrm{ro}}$ \\
\hline$\left(c, \varepsilon_{c}\right)=2^{\left(a, \varepsilon_{a}\right)}$ & $\varepsilon_{c}=\left[2^{a}+\varepsilon_{\mathrm{ro}}\right] \cdot\left[2^{\varepsilon_{a}}-1\right]+\varepsilon_{\mathrm{ro}}$ \\
\hline$\left(c, \varepsilon_{c}\right)=\cos \left(\theta, \varepsilon_{\theta}\right)$ & $\varepsilon_{c}=\left(|\sin (\theta)|_{\text {up }} \cdot \varepsilon_{\text {angle }}\right)+\varepsilon_{\text {ro }}+\frac{1}{2}\left(\varepsilon_{\text {angle }}\right)^{2}$ \\
\hline$\left(c, \varepsilon_{c}\right)=\sin \left(\theta, \varepsilon_{\theta}\right)$ & $\varepsilon_{c}=\left(|\cos (\theta)|_{\text {up }} \cdot \varepsilon_{\text {angle }}\right)+\varepsilon_{\text {ro }}+\frac{1}{2}\left(\varepsilon_{\text {angle }}\right)^{2}$ \\
\hline$\left(c, \varepsilon_{c}\right)=\arctan \left(a, \varepsilon_{a}\right)$ & $\varepsilon_{c}=\left\{\begin{array}{lr}\varepsilon_{a} /\left[1+\left(|a|-\varepsilon_{a}\right)^{2}\right]+\varepsilon_{\mathrm{ro}} & \text { if }|a|>\varepsilon_{a} \\
\varepsilon_{a}+\varepsilon_{\mathrm{ro}} & \text { if }|a| \leq \varepsilon_{a}\end{array}\right.$ \\
\hline
\end{tabular}

Notes:

1) The number $c$ was computed as the appropriate function of the argument(s), rounded nearest; $\varepsilon_{\text {ro }}$ denotes the roundoff error in $c$, taken to be the value of the least significant bit of $c$ (or the least representable number, if $c=0$ ). If an inadmissible argument for the function was included in the uncertainty interval the computer was instructed to abort the program and print an error message.

2) In computing the uncertainty radius $\varepsilon_{c}$, all rounding was carried out in such a way as to increase the final result. Sometimes rounding is explicitly indicated by a subscript "up" or "down".

$3)$ In the formulas for $\sin (\theta), \cos (\theta)$, the quantity $\varepsilon_{\text {angle }}$ denotes the sum of $\varepsilon_{\theta}$ and the error introduced by reducing $\theta$ to lie in the interval $[0, \pi / 4]$; the chip's actual output, given $\theta \in[0, \pi / 4]$, is a pair of numbers $x, y$ such that $y / x=\tan (\theta)$. These were assumed to be accurate within an error of 1 in the last bit, and $\sin (\theta), \cos (\theta)$ were computed from them (using interval arithmetic) using the formulas $\sin (\theta)=y /\left(x^{2}+y^{2}\right)^{1 / 2}, \cos (\theta)=x /\left(x^{2}+y^{2}\right)^{1 / 2}$.

certification. Finally, regarding the correct functioning of the machines: in the compilation of statistics about the zeros, the data was subjected to many consistency tests, and a few violations were found. All these were flagrantly unreasonable, and all but one could be traced to corrupted storage media. Upon recomputation, reasonable values were found in every case. These facts suggest that the computations were sound and that the data set is now clean.

\section{Proof that all zeros had beEn found}

A third program used a generalized Turing's criterion for Dirichlet $L$-series to show that all zeros up to a chosen height were on the critical line. Given a segment $\left(t_{1}, t_{2}\right)$ on the critical line, it combined a count of the zeros of $L(s, \chi)$ known to be in that segment, together with the existence of sufficiently long intervals about $t_{1}, t_{2}$ where the zeros are fairly regularly spaced, to rigorously prove that all zeros with $t \in\left(t_{1}, t_{2}\right)$ are on the line. This program took only a few minutes to run, for each modulus.

Our proof of the generalized Turing criterion is modelled on a proof by R. S. Lehman [8] of the Turing criterion for the Riemann Zeta-function; we will refer 
the reader to Lehman's paper for many details. We first develop the notation needed to state the result.

Suppose $L(s, \chi)$ is a primitive Dirichlet $L$-series with conductor $Q>1$. As in the introduction, put

$$
\theta(t, \chi)=(t / 2) \ln (Q / \pi)+\operatorname{Im}(\ln (\Gamma((s+\delta) / 2)))-\theta_{\chi} / 2 .
$$

If $L(s, \chi)$ has no zeros at height $t$ in the critical strip, define

$$
S(t, \chi)=\left.(1 / \pi) \cdot \operatorname{Im}(\ln (L(s, \chi)))\right|_{+\infty+i t} ^{1 / 2+i t},
$$

where the logarithm is determined by analytic continuation along the horizontal line at height $t$. In general, define $S(t, \chi)=\lim _{y \rightarrow t^{-}} S(y, \chi)$. By the functional equation, for $0<\sigma<1$

$$
L(\sigma+i t, \chi)=0 \quad \text { if and only if } L(1-\sigma+i t, \chi)=0 .
$$

Thus, $S(t, \chi)$ has an integer jump at the ordinate of each zero.

Turing's idea is very simple. Suppose $t_{1}<t_{2}$, and that $L(s, \chi)$ has no zeros at height $t_{1}$ or $t_{2}$. Using the argument principle, and integrating $\xi(s, \chi)$ around a box with corners at $1+\varepsilon+i t_{1}, 1+\varepsilon+i t_{2},-\varepsilon+i t_{2},-\varepsilon+i t_{1}$, one finds that

$$
\left.N(t, \chi)\right|_{t_{1}} ^{t_{2}}=\left.(1 / \pi) \theta(t, \chi)\right|_{t_{1}} ^{t_{2}}+\left.S(t, \chi)\right|_{t_{1}} ^{t_{2}}
$$

where $\left.N(t, \chi)\right|_{t_{1}} ^{t_{2}}$ is the number of zeros of $L(s, \chi)$ in the critical strip with ordinates between $t_{1}$ and $t_{2}$. If $S\left(t_{1}, \chi\right)$ and $S\left(t_{2}, \chi\right)$ can be found, then $\left.N(t, \chi)\right|_{t_{1}} ^{t_{2}}$ can be computed. If, further, it agrees with the number of zeros actually found on the critical line, then the ERH holds for $L(s, \chi)$ between those heights. Even if $t_{1}$ or $t_{2}$ is the ordinate of a zero, the ERH can still be established for $t$ in the open interval $\left(t_{1}, t_{2}\right)$ if $\lim _{t \rightarrow t_{1}^{+}} S(t, \chi)$ and $\lim _{t \rightarrow t_{2}^{-}} S(t, \chi)$ are known.

A value of $t$ for which $\theta(t, \chi) \in \pi \mathbb{Z}$ will be called a Gram point; we will write $g_{n}$ for any solution to $\theta\left(g_{n}, \chi\right)=n \pi$. Since

$$
Z(t, \chi)=e^{i \theta(t, \chi)} L\left(\frac{1}{2}+i t, \chi\right)
$$

is real-valued, $S(t, \chi)$ takes an integer value at each Gram point. It is easily shown that $\theta(t, \chi)$ is monotone increasing for $t \geq 20$, and it will be convenient to write

$$
T=E(t, \chi)=(1 / \pi) \theta(t, \chi),
$$

so that $T$, the coordinate on the "Gram point scale", is integer-valued at the points $g_{n}$. If $t \geq 20$ and $T=E(t, \chi)$, we will write $\mathscr{S}(T, \chi)$ for $S(t, \chi)$; thus, $\mathscr{S}(T, \chi)$ takes on an integer value for each $T \in \mathbb{Z}$. It also follows immediately from (3) that, between zeros of $L(s, \chi)$ on the Gram point scale, $\mathscr{S}(T, \chi)$ is monotone decreasing with slope -1 , and at the Gram height of each zero, it has an integer jump equal to the number of zeros.

The key to Turing's method is to determine $\mathscr{P}(T, \chi)$. Set

$$
B(Q, t)=.2929 \ln \left(\frac{Q T}{2 \pi}\right)+.0198\left[\ln \left(\frac{Q t}{2 \pi}\right)\right]^{2} \text {. }
$$


Theorem 1 (Generalized Turing-Lehman bound). Let $Q>1$ and suppose $50 \leq$ $t_{1}<t_{2} \leq t_{3} ;$ put $T_{1}=E\left(t_{1}, \chi\right), T_{2}=E\left(t_{2}, \chi\right)$. Then

$$
\left|\int_{T_{1}}^{T_{2}} \mathscr{S}(T, \chi) d T\right| \leq B\left(Q, t_{3}\right) \text {. }
$$

Theorem 1 will be proved later. In practice, the value of $B\left(Q, t_{3}\right)$ is quite modest; for example, if $Q=100$ and $t_{3}=2500$, then $B\left(Q, t_{3}\right) \approx 4.824$.

To apply this, suppose we have been computing zeros of $L(s, \chi)$ on the critical line, and that the number of zeros found to Gram height $T$ is $\widehat{\mathcal{N}}(T, \chi)$. Put

$$
\widehat{\mathscr{S}}(T, \chi)=\widehat{\mathscr{N}}(T, \chi)-T .
$$

Then $\widehat{\mathscr{S}}(T, \chi)$, for each $T$, differs from $\mathscr{S}(T, \chi)$ by an integer. Fix some point $T_{0}$, and let $\mathscr{S}\left(T_{0}, \chi\right)=\widehat{\mathscr{S}}\left(T_{0}, \chi\right)-\Delta_{0}$. If, as is most likely, no zeros have been missed, the number $\Delta_{0}$ can quickly be found by numerically integrating $\widehat{\mathscr{S}}(T, \chi)$ : suppose $T_{0}=E\left(t_{0}, \chi\right)$ and $T_{1}=E\left(t_{1}, \chi\right)$, with $50<t_{0}<t_{1}$; choose $t_{1}$ large enough that

$$
T_{1}-T_{0}>2 B=2 B\left(Q, t_{1}\right) .
$$

By Theorem 1,

$$
\left|\int_{T_{0}}^{T_{1}}\left(\widehat{\mathscr{S}}(T, \chi)-\Delta_{0}\right) d T\right|=\left|\int_{T_{0}}^{T_{1}} \mathscr{S}(T, \chi) d T\right| \leq B,
$$

so

$$
\left|\Delta_{0}-\frac{1}{T_{1}-T_{0}} \int_{T_{0}}^{T_{1}} \widehat{\mathscr{S}}(T, \chi) d T\right| \leq \frac{B}{T_{1}-T_{0}}<1 / 2,
$$

which determines the integer $\Delta_{0}$.

Even without assuming that all zeros have been located, it is easy to find $\Delta_{0}$ in practice. This is because $\mathscr{S}(T, \chi)$ jumps everywhere that $\widehat{\mathscr{S}}(T, \chi)$ jumps and possibly at other points as well, so for all $T>T_{0}$, we have $\mathscr{S}(T, \chi) \geq$ $\widehat{\mathscr{S}}(T, \chi)-\Delta_{0}$, while for all $T<T_{0}$, we have $\mathscr{S}(T, \chi) \leq \widehat{\mathscr{S}}(T, \chi)-\Delta_{0}$. Hence, if $T_{1}<T_{0}<T_{2}$, with $T_{1}, T_{2}$ as in Theorem 1 , and if $B=B\left(Q, t_{3}\right)$ as in Theorem 1, then

so

$$
\int_{T_{0}}^{T_{2}}\left(\widehat{\mathscr{S}}(T, \chi)-\Delta_{0}\right) d T \leq \int_{T_{0}}^{T_{2}} \mathscr{S}(T, \chi) d T \leq B
$$

$$
\Delta_{0} \geq \frac{1}{T_{2}-T_{0}}\left(\int_{T_{0}}^{T_{2}} \widehat{\mathscr{S}}(T, \chi) d T-B\right)
$$

similarly,

$$
\Delta_{0} \leq \frac{1}{T_{0}-T_{1}}\left(\int_{T_{1}}^{T_{0}} \widehat{\mathscr{S}}(T, \chi) d T+B\right) .
$$

If the inequalities (5) and (6) bracket a single integer, as is usually easy to achieve in practice, they determine $\Delta_{0}$. 
Suppose now that at two points $T_{1}<T_{2}$ we are able to determine integers $\Delta_{i}$ such that $\mathscr{S}\left(T_{i}, \chi\right)=\widehat{\mathscr{S}}\left(T_{i}, \chi\right)-\Delta_{i}$, and $\Delta_{1}=\Delta_{2}=\Delta$. Then $\mathscr{S}(T, \chi)=$ $\widehat{\mathscr{S}}(T, \chi)-\Delta$ for all $T \in\left[T_{1}, T_{2}\right]$. Returning to the variable $t$, we have found $S(t, \chi)$ and can apply (3) to verify the ERH.

There are many possible variations on this idea. In the program, the following result was used:

Proposition 3. Suppose points $50<t_{0}<t_{1}<\cdots<t_{m}$ are known such that the values $Z\left(t_{i}, \chi\right)$ are alternating in sign. Let $T_{i}=E\left(t_{i}, \chi\right), i=0, \ldots, m$, and put $B=B\left(Q, t_{m}\right)$. Then

$$
\begin{aligned}
& \mathscr{S}\left(T_{0}, \chi\right) \leq\left(T_{m}-T_{0}\right)^{-1}\left[B+\frac{1}{2}\left(T_{m}-T_{0}\right)^{2}-\sum_{i=1}^{m-1}\left(T_{m}-T_{i}\right)\right], \\
& \mathscr{S}\left(T_{m}, \chi\right) \geq\left(T_{m}-T_{0}\right)^{-1}\left[-B-\frac{1}{2}\left(T_{m}-T_{0}\right)^{2}+\sum_{i=1}^{m-1}\left(T_{i}-T_{0}\right)\right] .
\end{aligned}
$$

Proof. For the first formula, let $\check{\mathscr{S}}(T, \chi)$ be the function whose value at $T_{0}$ is $\mathscr{S}\left(T_{0}, \chi\right)$, which has slope -1 on each interval $\left(T_{i-1}, T_{i}\right)$, and which jumps by 1 at each of the points $T_{1}, \ldots, T_{m}$. By hypothesis, $Z(t, \chi)$ has a zero in each interval $\left(t_{i-1}, t_{i}\right)$; hence $\mathscr{S}(T, \chi)$ has a jump in each interval $\left(T_{i-1}, T_{i}\right)$, and so $\mathscr{S}(T, \chi) \geq \check{\mathscr{S}}(T, \chi)$ for all $T \in\left[T_{0}, T_{m}\right]$. The formula arises by using the inequality

$$
\int_{T_{0}}^{T_{m}} \check{\mathscr{S}}(T, \chi) d T \leq \int_{T_{0}}^{T_{m}} \mathscr{S}(T, \chi) d T \leq B,
$$

evaluating the first integral, and solving for $\mathscr{S}\left(T_{0}, \chi\right)$. The second formula is proved in a similar way, but working from the right end of $\left[T_{0}, T_{m}\right]$ to the left.

In applying Proposition 3, the program took the points $t_{i}$ to be the successive " $V$-points" intermediate to the zeros, found in Phase II, where the sign of $Z(t, \chi)$ had been rigorously determined. The function $\Delta(T)=\widehat{\mathscr{S}}(T, \chi)-$ $\mathscr{S}(T, \chi)$ is nonincreasing and integer-valued for all $T$. If all the zeros had been found, $\Delta(T)$ would be a constant $\Delta$; as indicated above, it is easy to guess $\Delta$. To show $\Delta(T) \equiv \Delta$, the program first took $t_{0}$ to be the smallest $V$-point with $t_{0}>50$, and examined successive $V$-points $t_{1}, t_{2}, \ldots$ until it found a point $t_{m}$ such that the lower bound for $\mathscr{S}\left(T_{m}, \chi\right)$ given in Proposition 3 was strong enough to show $\Delta\left(T_{m}\right)<\Delta+1$. It then went to the top of the file and (starting notations anew) took $t_{m}$ to be the last $V$-point, then worked its way down until a point $t_{0}$ was reached such that the upper bound for $\mathscr{S}\left(T_{0}, \chi\right)$ was strong enough to show $\Delta\left(T_{0}\right)>\Delta-1$. Although there was no guarantee that this procedure would succeed, it always did. Letting $t_{\#}(\chi), t^{\#}(\chi)$ denote the two $V$-points where the bounds were established, it follows that for $T=E(t, \chi)$ with $t_{\#}(\chi)<t<t^{\#}(\chi)$, one has $\mathscr{S}(T, \chi)=\widehat{\mathscr{S}}(T, \chi)-\Delta$.

This would have sufficed to prove the ERH for $L(s, \chi)$ on $\left(t_{\#}(\chi), t^{\#}(\chi)\right)$, but it would not establish it for $0 \leq t<t_{\#}(\chi)$. To do so, the program linked the lists of zeros for $L(s, \chi)$ and $L(s, \bar{\chi})$ and applied (3) to compute $N(t, \chi)$ 
at $t=-t^{\#}(\bar{\chi})$, using that:

$$
\begin{array}{ll}
\theta(-t, \chi)=-\theta(t, \bar{\chi}) & \text { provided }-\theta_{\chi}=\theta_{\bar{\chi}} \text { (which was checked), } \\
S(-t, \chi)=-S(t, \bar{\chi}) & \text { for } t \text { which are not the ordinate of a zero. }
\end{array}
$$

By comparing the known count of zeros on the critical line with the bound for the number of zeros in the critical strip given by (3), the program established the ERH for $L(s, \chi)$ on the interval $\left(-t^{\#}(\bar{\chi}), t^{\#}(\chi)\right)$. The result is rigorous, since the only values of $Z(t, \chi)$ used were ones computed using interval arithmetic.

We now turn to the proof of Theorem 1. If $T_{1}=E\left(t_{1}, \chi\right)$ and $T_{2}=E\left(t_{2}, \chi\right)$ with $50<t_{1}<t_{2}$, then

$$
\begin{aligned}
\int_{T_{1}}^{T_{2}} S(T, \chi) d T & =\int_{t_{1}}^{t_{2}} S(t, \chi) \cdot E^{\prime}(t, \chi) d t \\
& =\left.E^{\prime}(t, \chi) \cdot S_{1}(t, \chi)\right|_{t_{1}} ^{t_{2}}-\int_{t_{1}}^{t_{2}} S_{1}(t, \chi) \cdot E^{\prime \prime}(t, \chi) d t
\end{aligned}
$$

where

$$
S_{1}(t, \chi)=\int_{t_{2}}^{t} S(y, \chi) d y .
$$

(We have arranged that $S_{1}\left(t_{2}, \chi\right)=0$.) Using Stirling's formula, one easily finds that, for $t \geq 20, E^{\prime}(t, \chi)$ and $E^{\prime \prime}(t, \chi)$ are both positive; hence

$$
\begin{aligned}
\left|\int_{T_{1}}^{T_{2}} \mathscr{S}(T, \chi) d T\right| \leq & \left|S_{1}\left(t_{1}, \chi\right)\right| \cdot E^{\prime}\left(t_{1}, \chi\right) \\
& +\left[\max _{t_{1} \leq t \leq t_{2}}\left|S_{1}(t, \chi)\right|\right] \cdot\left[\left.E^{\prime}(t, \chi)\right|_{t_{1}} ^{t_{2}}\right] \\
\leq & \max _{t_{1} \leq t \leq t_{2}}\left|S_{1}(t, \chi)\right| \cdot E^{\prime}\left(t_{2}, \chi\right) .
\end{aligned}
$$

Again by Stirling's formula (see [8, pp. 312-313] for a similar result) one finds that for $t \geq 50$

$$
E^{\prime}(t, \chi)=\frac{1}{2 \pi} \ln \left(\frac{Q t}{2 \pi}\right)+\Theta\left(\frac{.0072}{t^{2}}\right) \leq 0.1592 \ln \left(\frac{Q t}{2 \pi}\right),
$$

where $\boldsymbol{\Theta}(f(t))$ means a function $g(t)$ satisfying $-f(t) \leq g(t) \leq f(t)$. Below we will prove

Theorem 2. For $50<t_{1}<t_{2}$,

$$
\left|\int_{t_{1}}^{t_{2}} S(t, \chi) d t\right| \leq 1.8397+.1242 \ln \left(\frac{Q t_{2}}{2 \pi}\right) .
$$

Inserting these estimates in (8), one obtains Theorem 1.

Thus Theorem 1 is reduced to Theorem 2 . Theorem 2 follows immediately from the following pair of results:

Lemma 3 (Littlewood's Theorem for Dirichlet $L$-series). If $t_{1}, t_{2}$ are not the ordinates of zeros of $L(s, \chi)$, then, writing $s=\sigma+i$, one has

$$
\int_{t_{1}}^{t_{2}} S(t, \chi) d t=(1 / \pi) \int_{1 / 2+i t_{2}}^{+\infty+i t_{2}} \ln |L(s, \chi)| d \sigma-(1 / \pi) \int_{1 / 2+i t_{1}}^{+\infty+i t_{1}} \ln |L(s, \chi)| d \sigma,
$$

where the integrals are taken over the horizontal rays indicated.

The proof is the same as that for $\zeta(s)$ : see [4, pp. 190-192]. 
Theorem 3. For $t \geq 50$,

$$
-3.4507-.24 \ln \left(\frac{Q t}{2 \pi}\right) \leq \int_{1 / 2+i t}^{+\infty+i t} \ln |L(s, \chi)| d \sigma \leq 2.3288+.15 \ln \left(\frac{Q t}{2 \pi}\right) \text {. }
$$

For the proof of the upper bound in Theorem 3 we will need

Lemma 4 (Rademacher). Suppose $0<\eta<0.5$. Then for $-\eta \leq \sigma \leq 1+\eta$, for all moduli $Q>1$, and for all primitive characters $\chi$ with modulus $Q$,

$$
|L(s, \chi)| \leq\left(\frac{Q|1+s|}{2 \pi}\right)^{(1+\eta-\sigma) / 2} \cdot \zeta(1+\eta) .
$$

Proof. See [19, Theorem 3, p. 199]. The author would like to thank O. Ramare for pointing out this result, which enabled him to improve the constants in Theorems 1,2 , and 3 .

We now prove the upper bound in Theorem 3. Taking $\eta=0.25$, we obtain from Lemma 4

$$
\ln |L(s, \chi)| \leq(5 / 8-\sigma / 2) \cdot[\ln (Q|1+s| /(2 \pi))]+\ln (\zeta(1.25)) .
$$

By [8, p. 305], $\zeta(1.25) \leq 4.596$; also, for $\frac{1}{2} \leq \sigma \leq 1.25$ and $t \geq 50$, $\ln (|1+s| / t) \leq .0011$. Finally, [8, p. 305] gives the bound

$$
\int_{1.25+i t}^{+\infty+i t} \ln |\zeta(\sigma)| d \sigma \leq 1.184
$$

Combining these, we find

$$
\begin{aligned}
\int_{1 / 2+i t}^{+\infty+i t} \ln |L(s, \chi)| d \sigma & =\int_{1 / 2+i t}^{1.25+i t} \ln |L(s, \chi)| d \sigma+\int_{1.25+i t}^{+\infty+i t} \ln |L(s, \chi)| d \sigma \\
& \leq \int_{0.5}^{1.25}(.625-0.5 \sigma) \ln \left(\frac{Q t}{2 \pi}\right)+1.5263 d \sigma+1.184 \\
& \leq .1407 \ln \left(\frac{Q t}{2 \pi}\right)+2.3288
\end{aligned}
$$

as claimed.

For the lower bound in Theorem 3, we begin by writing

$$
\begin{aligned}
\int_{1 / 2+i t}^{+\infty+i t} & \ln |L(s, \chi)| d \sigma \\
= & \int_{1 / 2+i t}^{+\infty+i t} \ln \left|\frac{L(s, \chi) L(s+2, \chi)}{L(s+1, \chi)^{2}}\right| d \sigma \\
& +\int_{1.5+i t}^{+\infty+i t} \ln |L(s, \chi)| d \sigma+\int_{1.5+i t}^{2.5+i t} \ln |L(s, \chi)| d \sigma .
\end{aligned}
$$

Now, for $\sigma>1, \ln |L(s, \chi)|=-\sum_{p} \ln \left|1-\chi(p) p^{-s}\right|$. For each $p$,

$$
-\ln \left|1-\chi(p) p^{-s}\right| \geq-\ln \left|1+p^{-\sigma}\right|=-\ln \left|1-p^{-2 \sigma}\right|+\ln \left|1-p^{-\sigma}\right| ;
$$


hence $\ln |L(s, \chi)| \geq \ln |\zeta(2 \sigma)|-\ln |\zeta(\sigma)|$. Using these bounds in the last two terms and changing variables as needed, we find

$$
\begin{gathered}
\int_{1.5+i t}^{+\infty+i t} \ln |L(s, \chi)| d \sigma \geq \frac{1}{2} \int_{3}^{\infty} \ln |\zeta(\sigma)| d \sigma-\int_{1.5}^{\infty} \ln |\zeta(\sigma)| d \sigma, \\
\int_{1.5+i t}^{2.5+i t} \ln |L(s, \chi)| d \sigma \geq \frac{1}{2} \int_{3}^{5} \ln |\zeta(\sigma)| d \sigma-\int_{1.5}^{2.5} \ln |\zeta(\sigma)| d \sigma .
\end{gathered}
$$

By $[8$, p. 306],

$$
\begin{gathered}
\int_{1.5}^{2.5} \ln |\zeta(\sigma)| d \sigma<.5382, \quad \int_{2.5}^{\infty} \ln |\zeta(\sigma)| d \sigma<.3445 \\
\int_{5}^{\infty} \ln |\zeta(\sigma)| d \sigma<.0495, \quad \int_{3}^{\infty} \ln |\zeta(\sigma)| d \sigma>.2274 \\
\int_{3}^{5} \ln |\zeta(\sigma)| d \sigma>.1779
\end{gathered}
$$

(the last inequality follows from the previous two). On the other hand,

$$
\begin{aligned}
& \int_{1 / 2+i t}^{+\infty+i t} \ln \left|\frac{L(s, \chi) L(s+2, \chi)}{L(s+1, \chi)^{2}}\right| d \sigma \\
& \quad=\lim _{N \rightarrow \infty}\left(\int_{1 / 2+i t}^{N+i t} \ln \left|\frac{L(s, \chi)}{L(s+1, \chi)}\right|-\ln \left|\frac{L(s+1, \chi)}{L(s+2, \chi)}\right| d \sigma\right) \\
& \quad=\lim _{N \rightarrow \infty}\left(\int_{1 / 2+i t}^{1.5+i t} \ln \left|\frac{L(s, \chi)}{L(s+1, \chi)}\right| d \sigma-\int_{N+i t}^{N+1+i t} \ln \left|\frac{L(s, \chi)}{L(s+1, \chi)}\right| d \sigma\right) \\
& \quad=\int_{1 / 2+i t}^{1.5+i t} \ln \left|\frac{L(s, \chi)}{L(s+1), \chi}\right| d \sigma .
\end{aligned}
$$

Inserting (14), (15), (16) in (13), one obtains

$$
\int_{1 / 2+i t}^{+\infty+i t} \ln |L(s, \chi)| d \sigma>\int_{1 / 2+i t}^{1.5+i t} \ln \left|\frac{L(s, \chi)}{L(s+1, \chi)}\right| d \sigma-1.2183 .
$$

Next we adduce the Weierstrass product

$$
(Q / \pi)^{s} \cdot \Gamma((s+\delta) / 2) \cdot L(s, \chi)=\xi(s, \chi)=e^{A+B s} \cdot \prod_{\rho}(1-s / \rho) e^{s / \rho}
$$

(cf. [2, p. 82]: the product is taken over the nontrivial zeros of $L(s, \chi)$ and converges absolutely and uniformly on compact subsets of $\mathbb{C} ; A$ and $B$ depend on $\chi)$. This yields

$$
\begin{aligned}
\ln \left|\frac{L(s, \chi)}{L(s+1, \chi)}\right|= & -\operatorname{Re}(B)+\frac{1}{2} \ln (Q / \pi)+\ln \left|\frac{\Gamma((s+1+\delta) / 2)}{\Gamma((s+\delta) / 2)}\right| \\
& +\sum_{\rho}\left(\ln \left|\frac{1-s / \rho}{1-(s+1) / \rho}\right|-\operatorname{Re}(1 / \rho)\right) .
\end{aligned}
$$

However, by [2, p. 83],

$$
\operatorname{Re}(B)=-\sum_{\rho} \operatorname{Re}(1 / \rho)
$$


where the sum converges absolutely, so the dependence of (19) on $\operatorname{Re}(B)$ and the $\operatorname{Re}(1 / \rho)$ cancels out. Further, the sum

$$
\sum_{\rho} \ln \left|\frac{1-s / \rho}{1-(s+1) / \rho}\right|
$$

converges absolutely and uniformly on compact subsets of $\mathbb{C} \backslash\{$ all $\rho, \rho-1\}$.

Now clearly,

$$
\int_{1 / 2}^{1.5} \frac{1}{2} \ln (Q / \pi) d \sigma=\frac{1}{2} \ln (Q / \pi),
$$

and by using the mean value theorem for integrals, as in [8, p. 311], one obtains

$$
\int_{1 / 2+i t}^{1.5+i t} \ln \left|\frac{\Gamma((s+1+\delta) / 2)}{\Gamma((s+\delta) / 2)}\right| d \sigma=\frac{1}{2} \operatorname{Re}\left(\frac{\Gamma^{\prime}(\tau+i t / 2)}{\Gamma(\tau+i t / 2)}\right)
$$

for some $\tau \in\left[\frac{1}{2}(.5+\delta), \frac{1}{2}(2.5+\delta)\right] \subset[.25,1.75]$. According to [8, Lemma 8, p. 308], if $\operatorname{Re}(z)>0$, then

$$
\frac{\Gamma^{\prime}(z)}{\Gamma(z)}=\ln (z)-\frac{1}{2 z}+\Theta\left(\frac{2}{\pi^{2}\left|\operatorname{Im}(z)^{2}-\operatorname{Re}(z)^{2}\right|}\right) .
$$

Applying this with $z=\tau+i t / 2$, and using $.25 \leq \tau \leq 1.75, t \geq 50$, we find

$$
\int_{1 / 2+i t}^{1.5+i t} \ln \left|\frac{\Gamma((s+1+\delta) / 2)}{\Gamma((s+\delta) / 2)}\right| d \sigma \geq \frac{1}{2} \ln (t / 2)-.0018 \text {. }
$$

Finally, consider

$$
\int_{1 / 2+i t}^{1.5+i t} \sum_{\rho} \ln \left|\frac{1-s / \rho}{1-(s+1) / \rho}\right| d \sigma=\sum_{\rho} \int_{1 / 2+i t}^{1.5+i t} \ln \left|\frac{s-\rho}{s+1-\rho}\right| d \sigma .
$$

(The interchange of sum and integral is justified by uniform convergence.) By [8, Lemma 7, p. 307], if $\operatorname{Re}(a) \geq 0, a \neq 0$, then

$$
\int_{a-1}^{a} \ln |z /(z+1)| d x \geq-1.48 \cdot \operatorname{Re}(1 / a) \text {. }
$$

Hence,

$$
\sum_{\rho} \int_{1 / 2+i t}^{1.5+i t} \ln \left|\frac{s-\rho}{s+1-\rho}\right| d \sigma \geq-1.48 \sum_{\rho} \operatorname{Re}\left(\frac{1}{1.5+i t-\rho}\right) .
$$

To simplify this, logarithmically differentiate (18), take real parts and use (20) again; this gives

$$
\sum_{\rho} \operatorname{Re}\left(\frac{1}{s-\rho}\right)=\frac{1}{2} \ln (Q / \pi)+\frac{1}{2} \operatorname{Re}\left(\frac{\Gamma^{\prime}((s+\delta) / 2)}{\Gamma((s+\delta) / 2)}\right)+\operatorname{Re}\left(\frac{L^{\prime}(s, \chi)}{L(s, \chi)}\right)
$$

Taking $s=1.5+i t$ and using (28) in (27) yields

$$
\begin{aligned}
& \sum_{\rho} \int_{1 / 2+i t}^{1.5+i t} \ln \left|\frac{s-\rho}{s+1-\rho}\right| d \sigma \\
& \quad \geq-1.48\left(\frac{1}{2} \ln (Q / \pi)+\frac{1}{2} \operatorname{Re}\left(\frac{\Gamma^{\prime}\left(\frac{3}{4}+\frac{1}{2} \delta+i t\right)}{\Gamma\left(\frac{3}{4}+\frac{1}{2} \delta+i t\right)}\right)+\operatorname{Re}\left(\frac{L^{\prime}(1.5+i t, \chi)}{L(1.5+i t, \chi)}\right)\right) .
\end{aligned}
$$


Using (23) once more, one finds that, since $t \geq 50$,

$$
\frac{1}{2} \operatorname{Re}\left(\frac{\Gamma^{\prime}\left(\frac{3}{4}+\frac{1}{2} \delta+i t\right)}{\Gamma\left(\frac{3}{4}+\frac{1}{2} \delta+i t\right)}\right) \leq \frac{1}{2} \ln (t / 2)+.0011 .
$$

Also for $\sigma=\operatorname{Re}(s)>1$,

$$
\begin{aligned}
\operatorname{Re}\left(\frac{L^{\prime}(s, \chi)}{L(s, \chi)}\right) & =\operatorname{Re}\left(-\sum_{p} \frac{\chi(p) \ln (p)}{p^{s}-\chi(p)}\right) \\
& \leq-\sum_{p} \ln (p) /\left(p^{\sigma}-1\right)=-\zeta^{\prime}(\sigma) / \zeta(\sigma) .
\end{aligned}
$$

Now $[8$, p. 305$]$ gives

$$
-\zeta^{\prime}(1.5) / \zeta(1.5) \leq 1.506
$$

Thus, taking $s=1.5+i t$ in (31) and combining (17)-(32), one gets

$$
\begin{aligned}
\int_{1 / 2+i t}^{+\infty} & \ln |L(s, \chi)| d \sigma \\
\geq & \frac{1}{2} \ln (Q / \pi)+\left[\frac{1}{2} \ln (t / 2)-.0018\right]-1.2183 \\
& \quad-1.48\left[\frac{1}{2} \ln (Q / \pi)+\frac{1}{2} \ln (t / 2)+.0011+1.506\right] \\
\geq & -.24 \ln \left(\frac{Q t}{2 \pi}\right)-3.4507 .
\end{aligned}
$$

This is the desired lower bound in Theorem 3.

Note that the numbers in our lower bound are essentially the same as those in [8, Lemma 9, p. 309], while those in our upper bound are slightly worse than those in [8, Lemma 5, p. 307]; the reason is that our Lemma 4 is not as strong as $[8$, Lemma 4 , p. 306].

\section{Conclusions}

As a result of the computations described in the Introduction, and the discussion in $\S \S 1-3$, we have

Theorem 4. For all $Q \leq 13$, the ERH holds for all primitive Dirichlet $L$ functions $L(s, \chi)$ with modulus $Q$, for at least $|t| \leq 10000$. For all $Q \leq 72$, all composite $Q \leq 112$, and all $Q \in\{116,117,120,121,124,125,128,132$, $140,143,144,156,163,168,169,180,216,243,256,360,420,432\}$, that is, for the classes of moduli listed in the introduction, the ERH holds for all primitive $L(s, \chi)$ with modulus $Q$ for at least $|t| \leq 2500$. More precise results for individual moduli are given in Table 5.1 (in the Supplement section).

Disc files containing the following output are stored for each modulus and each $L(s, \chi)$ : the zeros of $L(s, \chi)$ and the points between them where $|L(s, \chi)|$ takes its maximum value, as well as $Z(t, \chi)$ at the maximum; " $V$-points" between each pair of zeros, the function values $Z(t, \chi)$ at the $V$-points, and error bounds for the values of those $Z(t, \chi)$. In addition, for each zero with $|t| \leq 50$, an additional $V$-point was recorded at a height $10^{-6}$ below the zero, to rigorously establish a lower bound for it. 
Section 5 contains several tables and figures summarizing the data. We will now discuss these. In this section, $T$ no longer denotes the coordinate on the Gram point scale, but is simply the usual height.

Table 5.1 gives summary statistics about the $L$-series. For each modulus $Q$, the table reports the extrema which occurred over all $L(s, \chi)$ with conductor $Q$. The columns of the table are:

\# L-SER: the number of primitive $L$-functions with conductor $Q$;

ERH HEIGHT: the height $T$ to which the ERH was proved for all $L(s, \chi)$ with conductor $Q$;

SUM $1 /|R|$ BOUND: a rigorous upper bound for the sum

$$
\sum_{\substack{\text { primitive } \\ \chi(\bmod Q)}}\left(\sum_{\substack{0<\rho<T \\ L(\rho, \chi)=0}} 1 /|\rho|\right)
$$

(this bound was computed by replacing each $\rho$ by the next smaller $V$-point below it, and at the end was rounded up to 3 decimal places);

LEAST ROOT: the smallest $\gamma \geq 0$ for which some $L\left(\frac{1}{2}+i \gamma, \chi\right)=0$;

LEAST $\mid$ MAX $\mid$ : the smallest maximum value of $|Z(t, \chi)|$ between zeros;

GREATEST $\mid$ MAX $\mid$ : the greatest maximum of $|Z(t, \chi)|$ between zeros;

GREATEST $|S(T)|$ : the greatest value of $|S(T, \chi)|$ found;

LEAST GAP, GREATEST GAP: the least gap and greatest gap between consecutive zeros (on the Gram point scale).

Table 5.2 gives detailed information about the 15 "champions" for various statistics. For each record, the table gives the conductor $Q$, the identification number $K$ for the $L$-series, the value of the statistic, and the location where it occurred. The statistics recorded are the LEAST and GREATEST gaps (on the Gram point scale); the LEAST CONSECUTIVE PAIRS of gaps; the LARGEST VALUES OF $|S(T, \chi)|$ (reported with their sign), and the LEAST and GREATEST MAXIMA of $|Z(t, \chi)|$. For all quantities except the GREATEST MAX, the values recorded are the extrema over all $Q, K$, with one possible report for each $(Q, K)$. For the GREATEST MAX, the values recorded are the extrema for all $Q, K$, with one possible report for each $Q$.

The most notable extremum is the very short gap of Gram length 0.001831 between zeros at heights 257.54604 and 257.54738 , with its corresponding small maximum of 0.000004 , for the $L$-series with conductor $Q=95$ and $K=8$ (the character determined by $\chi(77)=e(3 / 4), \chi(21)=e(5 / 18)$; here and below we write $e(w)$ for $\left.e^{2 \pi i w}\right)$. This point occurs at such a low height that the entire Riemann-Siegel formula is concentrated in the error term. Also notable is the short pair of consecutive gaps of total Gram length 0.212090 for $Q=121$, $K=99(\chi(112)=e(7 / 10), \chi(12)=e(1 / 11))$ between roots at 1766.606, 1766.627, and 1766.734. Four gaps of Gram length at least 3.0 were found, the longest being 3.1687 , for $Q=163, K=71(\chi(2)=e(43 / 162))$ between roots at 2376.696 and 2378.501 . The largest value of $S(t, \chi)$ encountered was -1.70084 , at height 1513.695 , for $Q=163, K=105(\chi(2)=e(145 / 162))$. The largest value of $|L(s, \chi)|$ found was 37.13567 at height 2182.831 , for $Q=163, K=74(\chi(2)=e(131 / 162))$.

Even a cursory scan of the Table 5.1 suggests that the $L$-series with composite conductor are much more constrained than those with prime conductor. Figure 
5.3 graphs the GREATEST |MAX $\mid$, GREATEST $|S(T)|$, LEAST GAP, and GREATEST GAP, to height 2500 , for all $L$-series with conductor $Q \leq 132$ dealt with in the study. These graphs show the range of values attained for a given $Q$, as well as the extrema. For the GREATEST $|\mathrm{MAX}|$ the dependence on the conductor is easiest to see. When the average GREATEST |MAX| is computed for each $Q$ at heights 100,500,1000, 2500, and fitted to various curves, a good fit is given by

$$
y=0.7275 \cdot Q^{-0.0472} \cdot\left(\frac{\varphi(Q)}{Q}\right)^{0.9441} \cdot\left(\ln \left(\frac{Q t}{2 \pi}\right)\right)^{1.4557}
$$

Note that $\varphi(Q) / Q$ depends only on the primes dividing $Q$.

Table 5.4 records most of the same statistics as Table 5.1 , but for individual $L$-series with conductor $Q \leq 13$. For each $K$, it gives the values, order, and sign of $\chi_{K}$, followed by the sequence number $\bar{K}$ for the character $\bar{\chi}_{K}$. The columns for the character values first list generators for $(\mathbb{Z} / Q \mathbb{Z})^{\times}$and below them the values: thus, for $Q=3$, the entries 2 and $1 / 2$ mean that $\chi(2)=e(1 / 2)$. The column "\# ROOTS" gives the number of roots of $L\left(s, \chi_{K}\right)$ with $0<\gamma<$ ERH HEIGHT: Table 5.5 reports the root counts and root sum bounds at the intermediate heights 100, 500,1000, 2500, 5000, 10000 for use in theorems of Rosser-Schoenfeld type.

Following the tables are several figures concerning the pair correlation conjecture and the GUE hypothesis.

Montgomery originally stated the pair correlation conjecture only for the Riemann zeta-function [15]. However, assuming the ERH, one expects it to hold for Dirichlet $L$-series as well. Given a primitive $L(s, \chi)$, let $E(t, \chi)=$ $(1 / \pi) \theta(t, \chi)$ be the coordinate on the Gram point scale, as in $\S 3$. List the zeros $\frac{1}{2}+i \gamma_{n}$ of $L(s, \chi)$ by increasing ordinates, starting at any fixed zero. The conjecture predicts that for $0<\Delta_{1}<\Delta_{2}$ one should have

$$
\begin{aligned}
\lim _{N \rightarrow \infty} & N^{-1} \cdot \#\left\{(n, k): 1 \leq n \leq N, E\left(\gamma_{n+k}, \chi\right)-E\left(\gamma_{n}, \chi\right) \in\left(\Delta_{1}, \Delta_{2}\right]\right\} \\
= & \int_{\Delta_{1}}^{\Delta_{2}}\left(1-\frac{\sin ^{2}(\pi x)}{(\pi x)^{2}}\right) d x .
\end{aligned}
$$

By tallying the counts on the left side, one can determine an "empirical pair correlation function". For the Riemann zeta function, Odlyzko [16] found excellent agreement between the empirical and theoretical pair correlation functions.

The GUE hypothesis, a much more far-reaching and speculative conjecture, says (loosely) that the zeros of $L(s, \chi)$ should behave statistically like eigenvalues of random Hermitian matrices chosen from the "Gaussian Unitary Ensemble" of mathematical physics. We will not explain the GUE hypothesis here, but refer the reader to [16] and the references therein. The GUE hypothesis implies the pair correlation conjecture, and makes many other predictions about the statistical properties of the zeros. Among these are the expected distribution functions for the gaps between $k$ consecutive zeros $(k=1,2, \ldots)$, and the assertion that the fractional parts $\left\langle E\left(\gamma_{n}, \chi\right)\right\rangle=E\left(\gamma_{n}, \chi\right)(\bmod 1)$ should be uniformly distributed in the unit interval. A major goal of Odlyzko's work [16] was to numerically test the GUE hypothesis for the Riemann zeta function, and again he found excellent agreement. 
The present study sought to examine these conjectures for $L$-series. To improve statistics, since only a few thousand zeros have been computed for each $L(s, \chi)$, the program averaged the empirical pair correlation functions for all $L(s, \chi)$ with a given conductor $Q$. For prime moduli, especially for large primes, the result was near Montgomery's pair correlation function. However, for composite moduli, especially for moduli divisible by 12 , the empirical pair correlation functions showed large oscillations. The functions varied from modulus to modulus, but for moduli with the same underlying prime factors they were almost identical.

In addition, density plots were computed for the roots (mod 1) on the Gram point scale (that is, density plots for the fractional parts $\left.\left\langle E\left(\gamma_{n}, \chi\right)\right\rangle\right)$. In all cases they were roughly sinusoidal in form, with a peak at $x=\frac{1}{2}$. However, they were fairly flat for prime moduli, more peaked for composite moduli, and quite peaked for moduli divisible by 12,60 , and 420 .

Figure 5.6 shows these results. The plots actually presented are the averages over all $L$-series whose conductor had a given set of primes as its support, but the plots for individual moduli, and even individual $L$-series, are very similar. For each set of underlying primes, the figure on the left is the distribution of the roots $(\bmod 1)$, and the figure on the right is the empirical pair correlation function. Plots are shown for moduli with supports $\{2\},\{3\},\{5\},\{7\}$, \{primes $\},\{2,3\},\{2,5\},\{3,5\},\{2,3,5\},\{2,3,5,7\}$. In addition, the GUE prediction and the empirical pair correlation function of $\zeta(s)$ (using zeros to height 10000) are given for comparison.

Figure 5.7 shows the pair correlation functions of individual $L$-series with $Q=9$ and 13 , to compare with the average pair correlation functions of those moduli (they are only a few of many that were plotted). These figures help justify our assertion that, apart from statistical scatter, all the $L$-functions with a given conductor $Q$ have the same pair correlation function.

Odlyzko has suggested an explanation for these phenomena. By the RiemannSiegel formula for Dirichlet $L$-series (see [23, Theorem 6]),

$$
Z(t, \chi)=2 \sum_{\substack{n=1 \\(n, Q)=1}}^{L} n^{-1 / 2} \cdot \cos (\theta(t, \chi)+\arg (\chi(n))-t \cdot \ln (n))+O\left(t^{-1 / 4}\right),
$$

where $L=Q\lfloor\sqrt{t /(2 \pi Q)}\rfloor$. Until $t$ is fairly large, the terms with small $n$ may be expected to dominate, and the $n=1$ term, $\cos ((\theta(t, \chi)))$, will be the most important. If $Q$ is divisible by small primes, then the terms where $n$ is divisible by those primes will be missing from the sum, and the $n=1$ term will dominate even longer. This suggests that for highly composite $Q$, the zeros will (at least initially) be much more regularly spaced than for prime $Q$, and that on the Gram point scale $(\bmod 1)$ the roots should cluster about $\frac{1}{2}$. This is precisely what is observed in Figure 5.6. Furthermore, if the density function for the roots $(\bmod 1)$ is $1-w \cdot \cos (2 \pi x)$, then (assuming the roots are randomly distributed (mod 1) subject to this density distribution), the pair correlation function would be $1+\frac{1}{2} w^{2} \cdot \cos (2 \pi x)$. In fact, the roots are not randomly distributed; neighboring roots tend to "repel" each other. However, for sufficiently long intervals the randomness assumption is more nearly valid. Thus, given a strongly peaked density function, one would expect that for large 
$x$ the pair correlation function should have regular oscillations. This is exactly what is seen in Figure 5.8. Finally, if the arguments in the cosine terms in the Riemann-Siegel formula are more or less "randomly" distributed $(\bmod 2 \pi)$, then one should see the same empirical pair correlation function (to a given height) for all $L$-series with the same conductor, as observed in Figure 5.6.

For large $t$, the dominance of the small terms in the Riemann-Siegel formula should diminish. One would expect the roots to become more uniformly distributed $(\bmod 1)$, and the empirical pair corr ation functions to converge to Montgomery's pair correlation function. However, the convergence is likely to be slower than it is for the Riemann zeta function.

Figure 5.8 shows extended graphs of the pair correlation functions for $Q=13$ and $Q=180$. It can be seen that for the prime modulus $Q=13$, the function is fairly flat but has a long-term pattern of low-amplitude "beats", while for the highly composite modulus $Q=180$ there are regular oscillations of large amplitude, superimposed on a pattern of beats. These graphs are typical of many that were plotted. Figure 5.8 also shows modified Fourier transforms of these pair correlation functions. More precisely, the function plotted in the lower graph is $F_{Q}(\alpha, t)=1-\left(1-f_{Q}(x)\right)^{\wedge}$, where $f_{Q}(x)$ is the pair correlation function, and $h^{\wedge}(\alpha)=\int_{0}^{30} h(x) \cos (2 \pi x \alpha) d x$. Here, $t=10000$ for $Q=13$, and $t=2500$ for $Q=180$. If we define

$$
F_{\chi}(\alpha, t)=\frac{2 \pi}{t \ln (Q t / 2 \pi)} \sum_{\substack{0<\gamma \leq t \\ 0<\gamma^{\prime} \leq t}} \frac{4 t^{i \alpha\left(\gamma-\gamma^{\prime}\right)}}{1+\left(\gamma-\gamma^{\prime}\right)^{2}},
$$

where $\frac{1}{2}+i \gamma, \frac{1}{2}+i \gamma^{\prime}$, run over zeros of $L(s, \chi)$, then $F_{Q}(\alpha, t)$ is basically the average of the $F_{\chi}(\alpha, t)$ for all $\chi$ with conductor $Q$, omitting the terms with $\gamma=\gamma^{\prime}$. Both $F_{13}(\alpha, t)$ and $F_{180}(\alpha, t)$ have spikes just to the right of $\alpha=1$, corresponding to the periodic oscillations of the pair correlation functions. For the trivial character $\chi_{0}$, Montgomery [15] showed that

$$
F_{\chi_{0}}(\alpha, t)=(1+o(1)) t^{-2 \alpha} \ln (t)+\alpha+o(1) \quad \text { as } t \rightarrow \infty,
$$

uniformly for $\alpha \in[0,1]$. For $\alpha \geq 1$, he conjectured that as $t \rightarrow \infty$, $F_{\chi_{0}}(\alpha, t)=1+o(1)$, uniformly for $\alpha \in[a, b]$ with $1 \leq a \leq b<\infty$. (This is essentially equivalent to the pair correlation conjecture.) One expects the same results to hold for the $F_{\chi}(\alpha, t)$ with $\chi \neq \chi_{0}$. Figure 5.8 supports this, though it raises the possibility that the convergence may not be uniform near $\alpha=1$. However, Ozluk [17] has studied functions similar to the $F_{\chi}(\alpha, t)$ which are averages over all $Q$ and all $\chi$, and obtained some uniform convergence results for them.

Figure 5.9 examines the distribution of gaps (on a Gram point scale) between nearest and second-nearest neighbor zeros, in the light of GUE predictions. For each, plots of the distribution are drawn and the $k$ th-power moments of the distribution about its mean ( 1 or 2 respectively) are given. For the nearest neighbor distributions, $\log (T), 1 / T$ and $1 / T^{2}$ moments are given as well. (These are the same moments examined by Odlyzko for $\zeta(s)$.) The gap distributions are studied for $L$-series in three categories: those with moduli divisible by 12 , all moduli, and prime moduli. (In each case, the data presented are the averages over all $L$-series in the category.) In addition, the GUE predictions, kindly 
supplied by Odlyzko, are shown. It can be seen that in all cases the empirical distributions are more sharply peaked than the GUE predictions, with the prime moduli coming closest to the GUE. Since the heights in this study are so low $(\leq 10000)$, it is surprising that the distributions approach the GUE predictions as well as they do. With increasing $t$ one would hope for even better agreement.

Finally, it is generally believed that the zeros of distinct $L(s, \chi)$ should be statistically independent. Figures 5.10 and 5.11 examine this hypothesis for pairs of $L$-series and for multiple sets of $L$-series. Because it is easy to work out the GUE prediction for the distribution of gaps between consecutive zeros, the plots focus on that statistic. Let $W_{1}(x)$ be the GUE (Gram-scale) distribution for gaps between consecutive zeros of one $L$-series (shown at the bottom left of Figure 5.9). Let $\chi_{1}, \ldots, \chi_{n}$ be distinct, and put $L_{n}(s)=\prod_{i=1}^{n} L\left(s, \chi_{i}\right)$. Let the Gram scale for $L\left(s, \chi_{i}\right)$ be $E\left(t, \chi_{i}\right)$, and define the Gram scale for $L_{n}(s)$ to be

$$
E_{n}(t)=\sum_{i=1}^{n} E\left(t, \chi_{i}\right)
$$

Assuming (a) the ERH, (b) that the Gram scale gaps between consecutive zeros of each $L\left(s, \chi_{i}\right)$ have the distribution function $W_{1}(x)$, and (c) that the zeros of the $L\left(s, \chi_{i}\right)$ are statistically independent, then the Gram scale gaps between consecutive zeros of $L_{n}(s)$ will (asymptotically) have the distribution $W_{n}(x)=$ $\frac{1}{n} \mathscr{F}_{n}\left(\frac{1}{n} x\right)$, where

$$
\begin{aligned}
\mathscr{F}_{n}(x)= & W_{1}(x) \cdot\left(\int_{x}^{\infty}(u-x) W_{1}(u) d u\right)^{n-1} \\
& +(n-1) \cdot\left(\int_{x}^{\infty} W_{1}(u) d u\right)^{2} \cdot\left(\int_{x}^{\infty}(u-x) W_{1}(u) d u\right)^{n-2}
\end{aligned}
$$

Hejhal gives the case $n=2$ of this formula in [5, p. 1374]; however, he replaces the Gram scale coordinate $E_{2}(t)$ by $\frac{1}{2} E_{2}(t)$.

Figure 5.10 considers pairs of $L$-series. The first graph shows the average, over all $Q \leq 19$, and all pairs $\chi_{1} \neq \chi_{2}$ having the same conductor $Q$, of the empirical gap distributions for $L\left(s, \chi_{1}\right) L\left(s, \chi_{2}\right)$. It is in excellent agreement with the GUE prediction. The distributions for a number of individual products $L\left(s, \chi_{1}\right) L\left(s, \chi_{2}\right)$ are also shown (a few of many that were plotted). For the most part they are in good agreement with the GUE prediction: even for the Dedekind zeta function of $\mathbb{Q}(\sqrt{-420})$ the agreement is quite good, which is surprising, since to height 2500 there are only 1985 zeros of $\zeta(s)$, as opposed to 4387 zeros of the quadratic $L$-function $L\left(s, \chi_{420}\right)$.

The only obvious deviations from the GUE prediction occur for products $L\left(s, \chi_{1}\right) L\left(s, \chi_{2}\right)$ where both $\chi_{1}$ and $\chi_{2}$ have the same conductor $Q$, and $Q$ is divisible by 12 . However, these can be explained in terms of the very nonuniform distribution of the roots $(\bmod 1)$ for such $L$-series (see Figure 5.6), together with phase offsets between the Gram scales $E\left(t, \chi_{1}\right)$ and $E\left(t, \chi_{2}\right)$. One has

$$
E\left(t, \chi_{i}\right)=\frac{t}{2 \pi} \ln \left(\frac{Q t}{2 \pi}\right)-(-1)^{\delta_{t}} \cdot \frac{\operatorname{sign}(t)}{8} \cdot \frac{\theta_{\chi_{1}}}{2 \pi}+O(1 /|t|),
$$

where $\delta_{i}$ and $\theta_{\chi_{i}}$ are as in the functional equation of $L\left(s, \chi_{i}\right)$. It follows that 
$E\left(t, \chi_{1}\right)$ and $E\left(t, \chi_{2}\right)$ have an almost constant phase offset

$$
\Delta=\left[(-1)^{\delta_{1}}-(-1)^{\delta_{2}}\right] \cdot \frac{\operatorname{sign}(t)}{8}+(2 \pi)^{-1}\left(\theta_{\chi_{1}}-\theta_{\chi_{2}}\right)+O(1 /|t|) .
$$

If $\Delta$ is near 0 , then since the zeros of each $L\left(s, \chi_{i}\right)$ cluster near $\frac{1}{2}(\bmod 1)$ on their respective Gram point scales, the joint gap distribution for $L\left(s, \chi_{1}\right) L\left(s, \chi_{2}\right)$ should be skewed towards small gaps. On the other hand, if $\Delta$ is near 0.5 , then the joint gap distribution should be skewed towards gaps of length 1 . Exactly this behavior is seen for $Q=60$, as shown in Figure 5.10. There are three primitive characters with conductor $60: \chi_{1}$ and $\chi_{2}$ are conjugate complex characters of order 4 , and $\chi_{3}$ is the quadratic character. The phase offsets between the three pairs of Gram scales are $0.3238,0.0881$, and 0.4119 ; both in the joint gap distributions, and in the joint pair correlation functions, the expected skewing appears.

Figure 5.11 considers the joint gap distributions for three or more $L$-series. In general, there is good agreement between experiment and theory, though in some cases there may be a slight deficiency of very small gaps. The examples shown were chosen to include cases where one might look for correlation, if it were to appear: for example, the four characters of order 10 for $Q=11$ are Galois-conjugate, and the product of the corresponding $L\left(s, \chi_{i}\right)$ is the Artin $L$-series of a $\mathbb{Q}$-irreducible representation of $\mathrm{Gal}(\overline{\mathbb{Q}} / \mathbb{Q})$. It should be noted that as the number of $L$-series increases, the strength of the GUE prediction becomes weaker and weaker: Mehta and des Cloizeaux [14] have shown that as $n \rightarrow \infty$, under rather mild conditions on a distribution $W(x)$ (chiefly that $W(0)=0$ ), the $n$-fold joint distribution based on $W(x)$ approaches $y=e^{-x}$.

\section{ACKNOWLEDGMENTS}

The author would like to thank the following persons for their help in running our program: Red Alford, Tom Brahana, Kevin Clancey, Elliot Gootman, Peter Maddox, Marshall Saade, Susan Whitehead; Tony Chang, Moustapha Gad, Beata Hebda, Peotr Hebda, Jay Jung, Greg Kendall, Sheon Kim, Ed Kringle, Carol McDonald, Fola Parrish, Katy Tan, Chi Yip, and Abraham Xiong.

The author extends special thanks to Andrew Odlyzko for providing numerical GUE predictions, and for allowing his interpretation of the data in terms of the Riemann-Siegel formula to be published. Finally, he thanks Dennis Hejhal for making him aware of Siegel's paper [23], and Olivier Ramaré for pointing out the lemma of Rademacher used in $\S 3$.

\section{BIBLIOGRAPHY}

1. R. P. Brent, On the zeros of the Riemann zeta function in the critical strip, Math. Comp. 33 (1979), 1361-1372.

2. H. Davenport, Multiplicative number theory, 2nd ed. (revised by H. L. Montgomery), Graduate Texts in Math., vol. 74, Springer-Verlag, New York, Berlin, Heidelberg, 1980.

3. D. Davies and C. B. Haselgrove, The evaluation of Dirichlet L-functions, Proc. Roy. Soc. London Ser. A 264 (1961), 122-132.

4. H. M. Edwards, Riemann's zeta function, Academic Press, New York and London, 1974.

5. D. A. Hejhal, Epstein zeta functions and supercomputers, Internat. Congr. Math. (Berkeley, 1986), vol. II, Amer. Math. Soc., Providence, RI, 1987, pp. 1362-1384. 
6. Intel Corporation, $i A P X 86 / 88,186 / 188$ user's manual, Santa Clara, California, 1986.

7. K. Iwasawa, Lectures on p-adic L-functions, Ann. of Math. Stud., no. 74, Princeton Univ. Press, Princeton, NJ, 1972.

8. R. S. Lehman, On the distribution of the zeros of the Riemann zeta-function, Proc. London Math. Soc. (3) 20 (1970), 303-320.

9. D. H. Lehmer, On the roots of the Riemann zeta-function, Acta Math. 95 (1956), 291-298.

10. __ Extended computation of the Riemann zeta-function, Mathematica 3 (1956), 102-108.

11. J. van de Lune, H. J. J. te Riele, and D. T. Winter, On the zeros of the Riemann zeta function in the critical strip. IV, Math. Comp. 46 (1986), 667-681.

12. K. S. McCurley, Explicit estimates for the error term in the prime number theorem for arithmetic progressions, Math. Comp. 42 (1984), 265-285.

13. ___ Explicit estimates for $\theta(x ; 3, l)$ and $\psi(x ; 3, l)$, Math. Comp. 42 (1984), 287-296.

14. M. L. Mehta and J. des Cloizeaux, The probabilities for several consecutive eigenvalues of a random matrix, Indian J. Pure Appl. Math. 3 (1972), 329-351.

15. H. L. Montgomery, The pair correlation function of zeros of the zeta function, Analytic Number Theory (H. G. Diamond, ed.), Proc. Sympos. Pure Math., vol. 24, Amer. Math. Soc. Providence, RI, 1973, pp. 181-193.

16. A. M. Odlyzko, The $10^{20}$-th zero of the Riemann zeta function and 175 million of its neighbors (to appear).

17. A. E. Ozluk, On the pair correlation of zeros of Dirichlet L-functions, Proc. First Conf. Canadian Number Theory Assoc. (Banff, 1988), R. A. Mollin, ed., W. de Gruyter, Berlin, 1990.

18. W. H. Press, B. P. Flannery, S. A. Teukolsky, and W. T. Vetterling, Numerical recipes, The art of scientific computing, Cambridge Univ. Press, New York, 1986.

19. H. Rademacher, On the Phragmén-Lindelöf theorem and some applications, Math. Z. 72 (1959), 192-204.

20. O. Ramaré, Contribution au problème de Goldbach: tout entier supérieur a 1 est somme d'au plus 13 nombres premiers, Thesis, Université de Bordeaux I, 1991.

21. __ Primes in arithmetic progressions, submitted to Math. Comp.

22.

23. C. L. Siegel, Contribution to the theory of Dirichlet L-series and the Epstein zeta-functions, Ann. of Math. 44 (1943), 143-172 (= Gesammelte Abhandlungen, vol. II, no. 42, pp. 360-389).

24. R. Spira, Calculation of Dirichlet L-functions, Math. Comp. 23 (1969), 489-497.

Department of Mathematics, University of Georgia, Athens, Georgia 30602

E-mail address: rr@math.uga.edu 


\section{Supplement to}

\section{NUMERICAL COMPUTATIONS CONCERNING THE ERH}

\section{ROBERT RUMELY}

\begin{tabular}{|c|c|c|c|c|c|c|c|c|c|}
\hline \multicolumn{2}{|c|}{ Table 5.1 : } & \multicolumn{7}{|c|}{$\begin{array}{l}\text { STATISTICS ABOUT L-SERIES, BY MODULUS } \\
\end{array}$} & \multirow{3}{*}{$\begin{array}{l}\text { GREATE.ST } \\
\text { GAP }\end{array}$} \\
\hline & $*$ & ERH & $\operatorname{SLM} 1: \mathrm{R}$ & I.EAST & LEAST & GREATEST & GREATEST & LEAST & \\
\hline Q & L-SER & HE IGHT & BOLND & ROOT & MAX & MAX & $S(T)$ & GAP & \\
\hline & & & & 14.13473 & & & & & \\
\hline $\begin{array}{l}1 \\
3\end{array}$ & $\begin{array}{l}1 \\
1\end{array}$ & 9.021 & 4.322 & $\begin{array}{r}14.13473 \\
8.03974\end{array}$ & & 16.90615 & 1.44847 & 0.04210 & 3351 \\
\hline & 1 & 133.702 & 5.672 & $\begin{array}{l}8.0 \\
6.0\end{array}$ & & 5.04223 & 1.34760 & 0.05368 & 2.59871 \\
\hline 5 & $\begin{array}{l}1 \\
3\end{array}$ & 248.86 & 6.092 & & & 11.86942 & 1. 37010 & 0.03333 & 2.52035 \\
\hline 7 & $\begin{array}{l}3 \\
5\end{array}$ & 10072.973 & 19.100 & 4. 13290 & & 19.62612 & 1.48385 & 0.03512 & 2.62789 \\
\hline 8 & $\begin{array}{l}5 \\
2\end{array}$ & 10017.500 & 34.278 & 2. 50937 & 00316 & 23.50929 & 1.52513 & 0.01357 & 2.75 \\
\hline 9 & $\begin{array}{l}2 \\
4\end{array}$ & 10021.581 & 14.056 & 3.57615 & 0.002 & 13.59221 & 1.37 & 0.04961 & 2. 48191 \\
\hline 11 & 4 & 10157.746 & 28.91 & & & 939 & 1.47636 & 0.02545 & 2.70072 \\
\hline 12 & & 1 & & & & 27.66859 & 1.61 & 0.02522 & 1486 \\
\hline 13 & $\begin{array}{r}1 \\
11\end{array}$ & 026.176 & 7.573 & 3.8 & 0.001977 & 9.30459 & 1.28586 & 0.03 & 2.41170 \\
\hline 15 & 3 & $\begin{array}{r}10019.119 \\
2524.086\end{array}$ & 86.079 & 0.88396 & 0.0 & $28.54^{\circ}$ & 1.65258 & 0.00899 & 365 \\
\hline 16 & 4 & $\begin{array}{l}2524.086 \\
2520.832\end{array}$ & 17.654 & & & 3095 & 1.29729 & 0.02518 & 2.22265 \\
\hline 17 & 15 & $\begin{array}{l}.832 \\
8.166\end{array}$ & 4.15 & & & 749 & 1. 33572 & 0.03984 & 2.42998 \\
\hline 19 & 17 & $\begin{array}{l}2748.166 \\
2512.243\end{array}$ & 94.573 & 0.39131 & 0.000748 & 23.89895 & 1.6 & 81 & 2. 69313 \\
\hline 20 & 3 & & 107.915 & 0.01896 & 0.0 & 23.24676 & 1.57042 & 0.04142 & 2. 82688 \\
\hline 21 & 5 & $\begin{array}{l}865 \\
293\end{array}$ & 18.9 & & 6685 & 9.64142 & 1. 28374 & 0.05928 & 2.22985 \\
\hline 23 & 21 & $\begin{array}{l}2544.293 \\
2542.055\end{array}$ & 31.877 & 1.61202 & 0.001040 & 14.41247 & 1.37 & 2660 & 2.47479 \\
\hline & 2 & $\begin{array}{l}2542.055 \\
2612.653\end{array}$ & 138.599 & 0.59543 & 0.0 & 24.61406 & 1.5 & & 2.79958 \\
\hline 25 & 16 & $\begin{array}{l}2612.653 \\
2506.712\end{array}$ & 13.220 & 1.9 & & & 778 & 0.02606 & 2.15096 \\
\hline 27 & 12 & $\begin{array}{l}2506.712 \\
2541.274\end{array}$ & 106.934 & 0.39645 & 0.000123 & 21.44001 & 1.47188 & 0.01258 & 2.60182 \\
\hline 28 & 5 & $\begin{array}{l}2541.274 \\
2503.605\end{array}$ & 81.797 & 0.40516 & 0. & 17.4 & 1.47291 & 0.02043 & 2.65296 \\
\hline 29 & 27 & & 33.848 & 1.20418 & & 11. & 1.32 & & 2. 40888 \\
\hline 31 & 29 & $\begin{array}{l}2512.351 \\
2515.510\end{array}$ & 186.749 & 0.28629 & 0.001480 & 25.70754 & 1.60159 & 0.02479 & 2. 84417 \\
\hline 32 & 8 & $\begin{array}{l}2515.510 \\
2646.407\end{array}$ & 203.685 & 0.00694 & 00809 & 25.5 & 1.63640 & 0.02346 & 80325 \\
\hline 33 & 9 & $\begin{array}{l}2646.407 \\
2530.468\end{array}$ & 57.191 & 3600 & & 13. & 1.46624 & 0.0 & 2. 48466 \\
\hline 35 & 15 & $\begin{array}{l}2530.468 \\
2510.826\end{array}$ & 3.5 & & 538 & 16.73714 & 1.40489 & 0.02966 & 2.46203 \\
\hline 3 & 4 & $\begin{array}{l}2510.826 \\
2585.494\end{array}$ & 106.872 & 0.58660 & 0.000182 & 20.25735 & 1.43655 & 0.008 & 2.53116 \\
\hline 37 & 35 & $\begin{array}{l}585.494 \\
520.072\end{array}$ & 28.858 & 0.9 & 0.002588 & 9.34404 & 167 & 308 & .34660 \\
\hline 39 & 11 & $\begin{array}{l}2520.072 \\
2524.507\end{array}$ & 254.727 & & & 28.984 & 1.61969 & 0.03535 & 93011 \\
\hline 40 & 6 & $\begin{array}{l}2524.507 \\
2586.457\end{array}$ & 0.0 & 5151 & 0.001633 & 18.52665 & 1.41161 & 0.02675 & .58028 \\
\hline 41 & 39 & $\begin{array}{l}2586.457 \\
2505.993\end{array}$ & 44.257 & 176 & & 11.3 & & & 4113 \\
\hline 43 & 41 & 505.9 & 289.095 & & & 21 & 1.6 & 342 & 2.84475 \\
\hline 44 & 9 & 505.223 & 6.8 & 0.13837 & 0.000026 & 27.88252 & 1.59767 & 0.00472 & .83346 \\
\hline 45 & 12 & 135.143 & 67.753 & 2372 & 0.000830 & 12.58658 & & & .49687 \\
\hline 47 & 45 & $\begin{array}{l}17.289 \\
06.052\end{array}$ & 90.410 & 0.1 & & & & 16 & .49948 \\
\hline 48 & 4 & 506.0 & 342.626 & & & & 1.64085 & 0.01762 & 2.88328 \\
\hline 49 & 36 & $\begin{array}{l}2521.707 \\
2508.798\end{array}$ & 0.55 & & 0.006073 & 9.27006 & 1. 29900 & 0.04740 & .36446 \\
\hline 51 & 15 & 26.701 & $\begin{array}{l}275.753 \\
115.205\end{array}$ & 0.09468 & 0.000230 & 26.84519 & & & 4264 \\
\hline 52 & 11 & 3.8 & 115.2 & & & & & 985 & 2.56331 \\
\hline 53 & 51 & $\begin{array}{l}553.836 \\
548.480\end{array}$ & 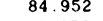 & & 0.000310 & 13.38493 & 1.41161 & 0.01423 & 2.46340 \\
\hline 55 & 2 & $\begin{array}{l}548.480 \\
509.998\end{array}$ & 398.252 & 0.00643 & 0.000399 & 29. & & & .96062 \\
\hline 56 & 10 & $\begin{array}{l}9.99 \\
6.78\end{array}$ & 210.645 & & & & & 0.03851 & .63395 \\
\hline 57 & 17 & $\begin{array}{l}16.7 \\
09.1\end{array}$ & & 0.52630 & 0.000730 & 12.70161 & 1.36402 & 0.02221 & .44271 \\
\hline 59 & 57 & $\begin{array}{l}2509.169 \\
2505.503\end{array}$ & 133.213 & 0.31033 & 0.000356 & 19.79805 & 1.55137 & & 2.75323 \\
\hline 60 & 3 & & 452.691 & 0.05165 & & 29.42993 & & & .94160 \\
\hline 61 & 59 & 3.720 & 3.76 & & & & & 0.05993 & 2. 24913 \\
\hline 63 & 20 & $\begin{array}{l}2512.627 \\
2503.432\end{array}$ & & & & 29.98239 & 1.68757 & 0.01400 & 3.03949 \\
\hline 64 & 16 & $\begin{array}{l}2503.432 \\
2508.451\end{array}$ & $\begin{array}{l}160.015 \\
128.467\end{array}$ & $\begin{array}{l}0.21101 \\
0.37696\end{array}$ & $\begin{array}{l}0.000553 \\
0.000095\end{array}$ & $\begin{array}{l}17.34584 \\
15.06637\end{array}$ & $\begin{array}{l}1.43459 \\
1.51673\end{array}$ & $\begin{array}{l}0.01819 \\
0.00851\end{array}$ & $\begin{array}{l}.51445 \\
.74952\end{array}$ \\
\hline 65 & 33 & 2516.155 & $\begin{array}{l}128.467 \\
265.591\end{array}$ & $\begin{array}{l}0.37696 \\
0.18416\end{array}$ & $\begin{array}{l}0.000095 \\
0.000867\end{array}$ & $\begin{array}{l}15.06637 \\
23.68456\end{array}$ & 73 & & $\begin{array}{l}.74952 \\
.72152\end{array}$ \\
\hline 67 & 65 & 2506.077 & 59 & 0.1 & & & & & $\begin{array}{l}72152 \\
96521\end{array}$ \\
\hline 68 & 15 & 2506.422 & 121.598 & 0.05346 & $\begin{array}{l}0.000263 \\
0.001380\end{array}$ & 14.93672 & $\begin{array}{l}1.75600 \\
1.47440\end{array}$ & $\begin{array}{r}0.01495 \\
0.03542\end{array}$ & $\begin{array}{l}96521 \\
55268\end{array}$ \\
\hline 69 & 21 & 2512006 & 170.883 & 0.22273 & 0.003124 & 19.76238 & 1.56791 & 0.04035 & 2. 65577 \\
\hline 71 & 69 & 2515.050 & 566.761 & 0.09859 & 0.000353 & 31.24616 & 1.68277 & 0.01463 & 2.97862 \\
\hline 72 & 8 & $261.4 \quad 933$ & 66.205 & $0 \quad 27916$ & 0.002330 & 9.81096 & 1. 398.38 & 0.03341 & 2.47542 \\
\hline
\end{tabular}




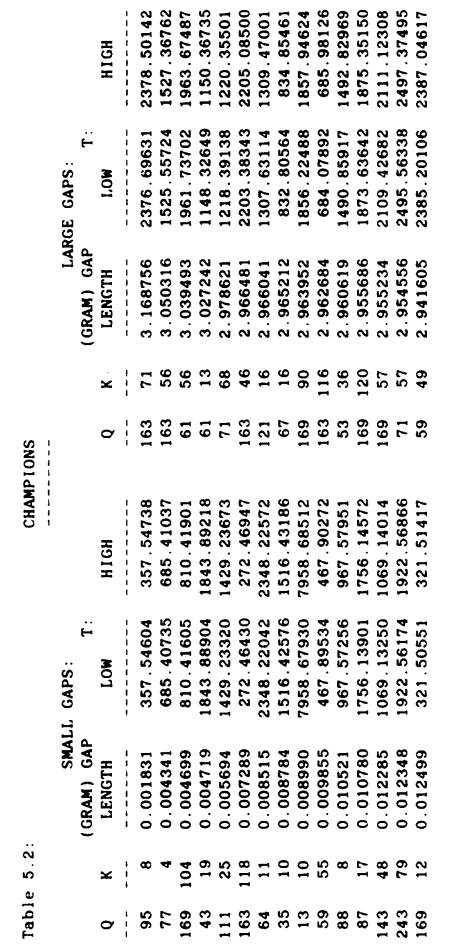

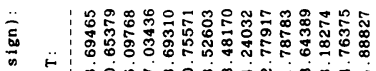

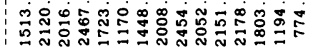

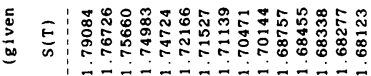

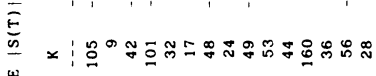

崖。|:

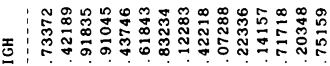

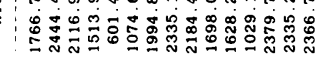

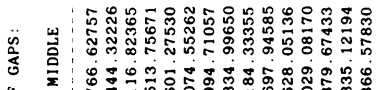

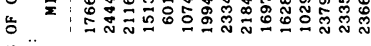

范

w

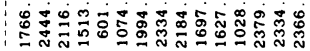

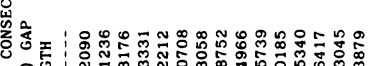
Ơ 00000000000000

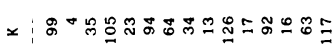

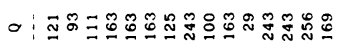

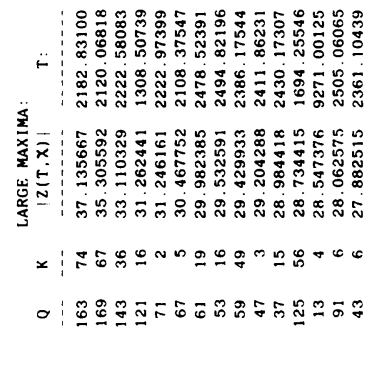

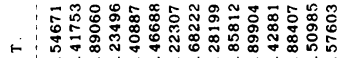

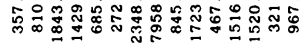

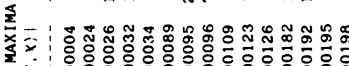

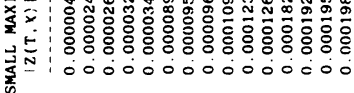

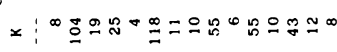
○

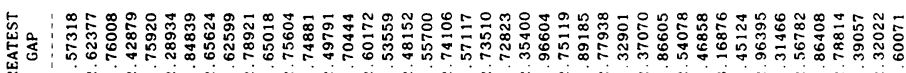

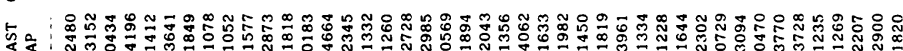

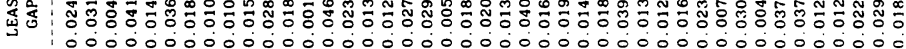

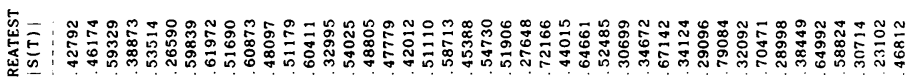

(1)

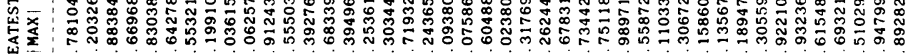

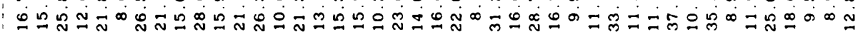

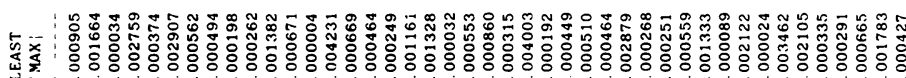

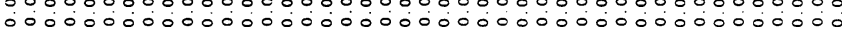

น 0000000000000000000000000000000000000000000

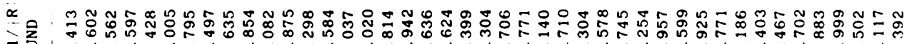

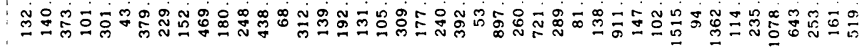

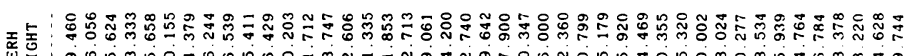
รัง

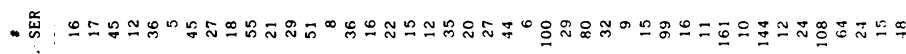



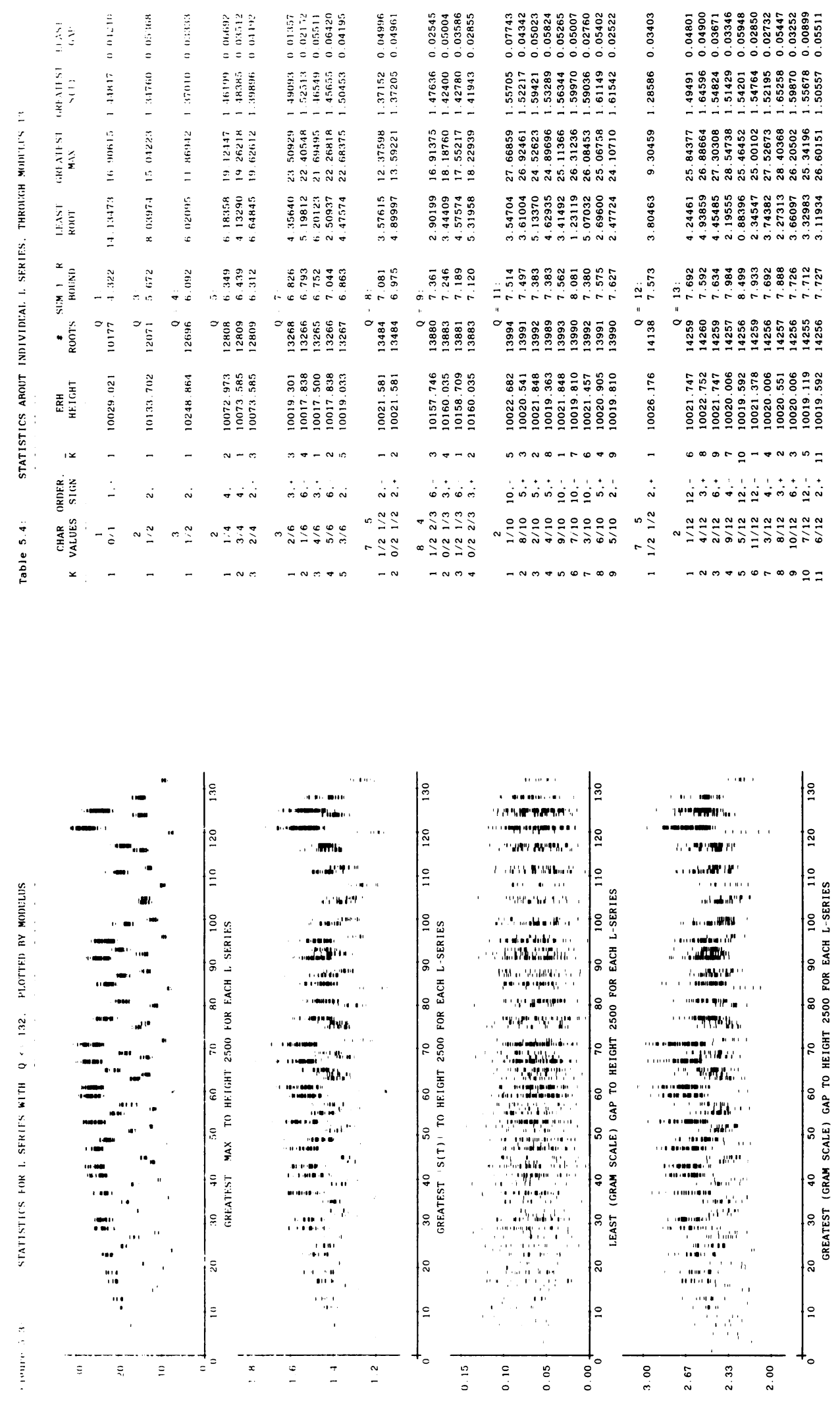


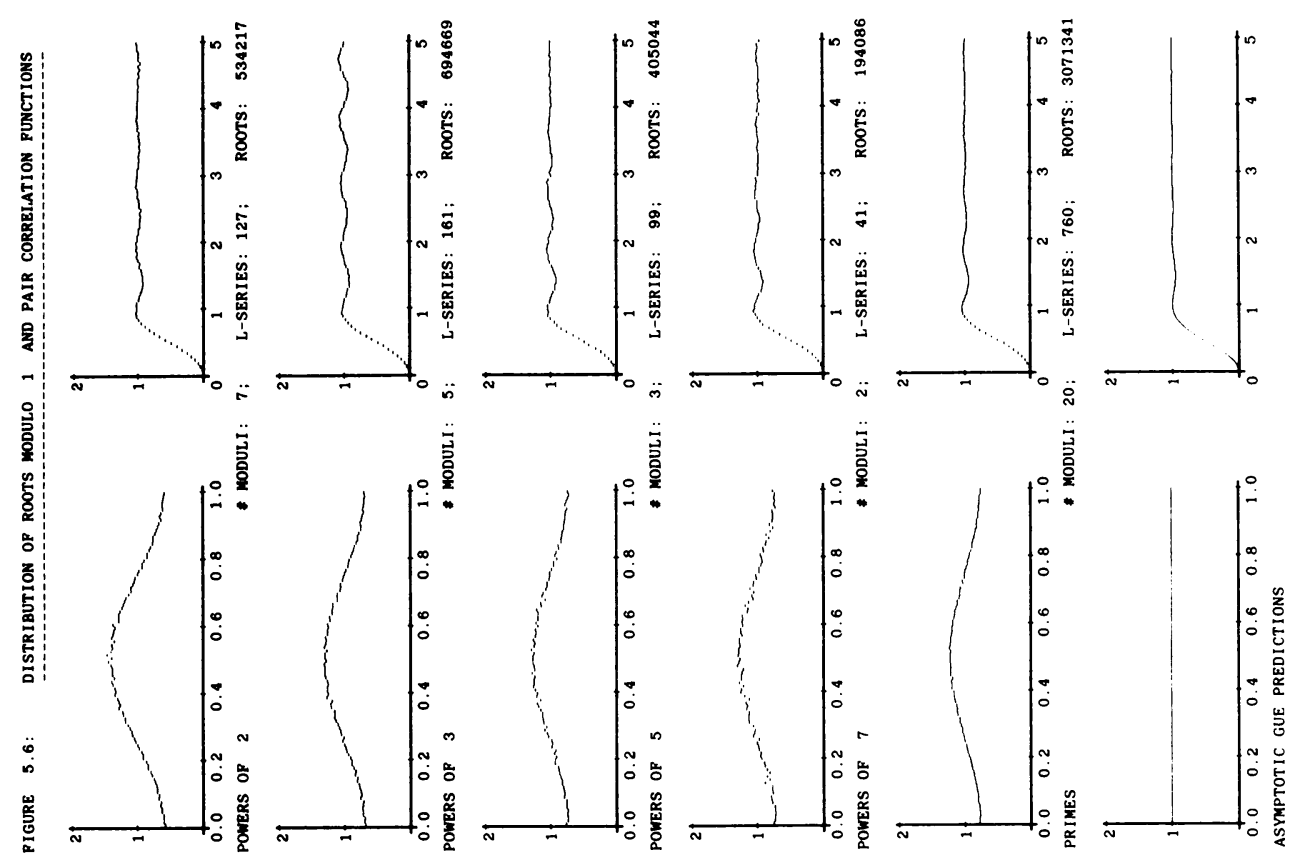

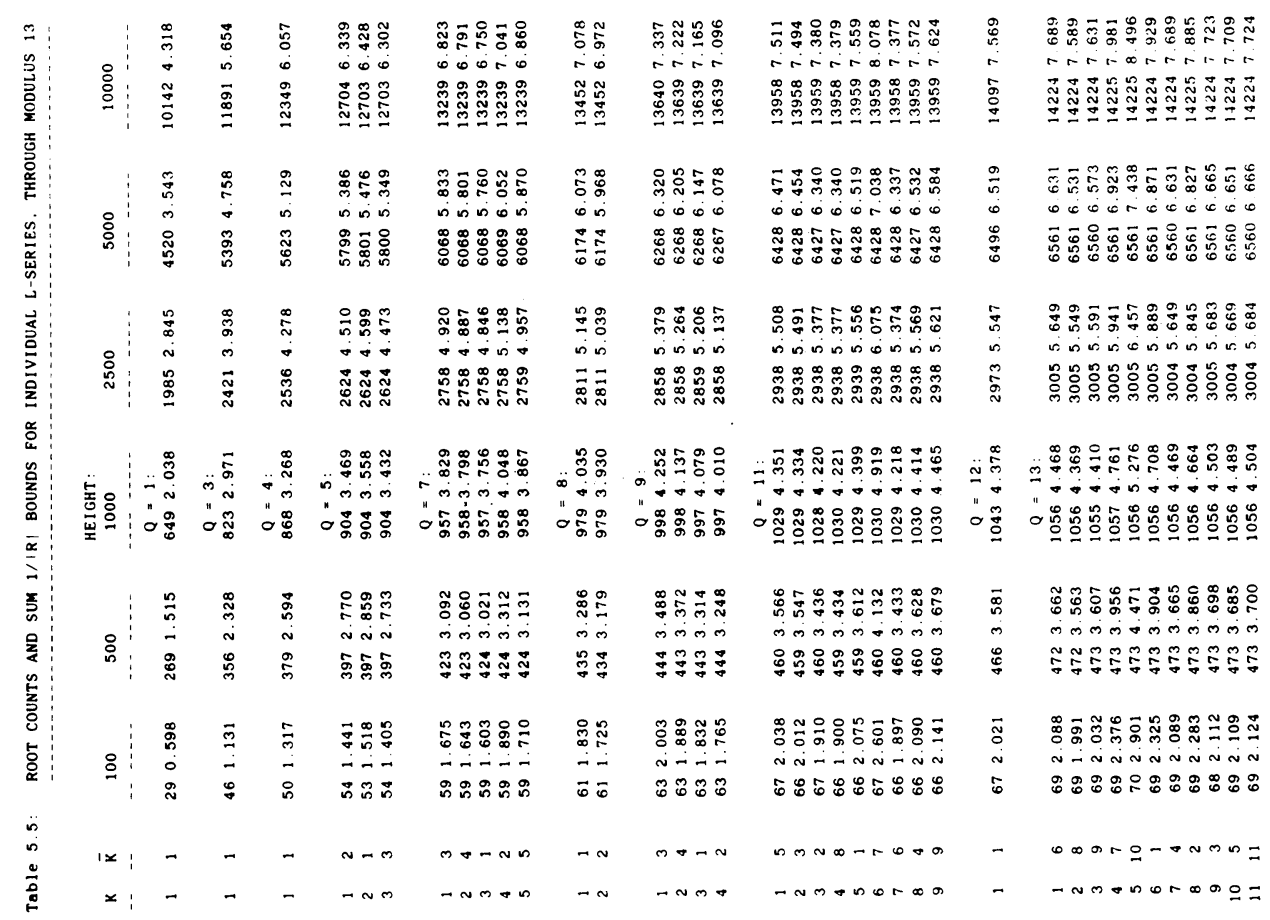



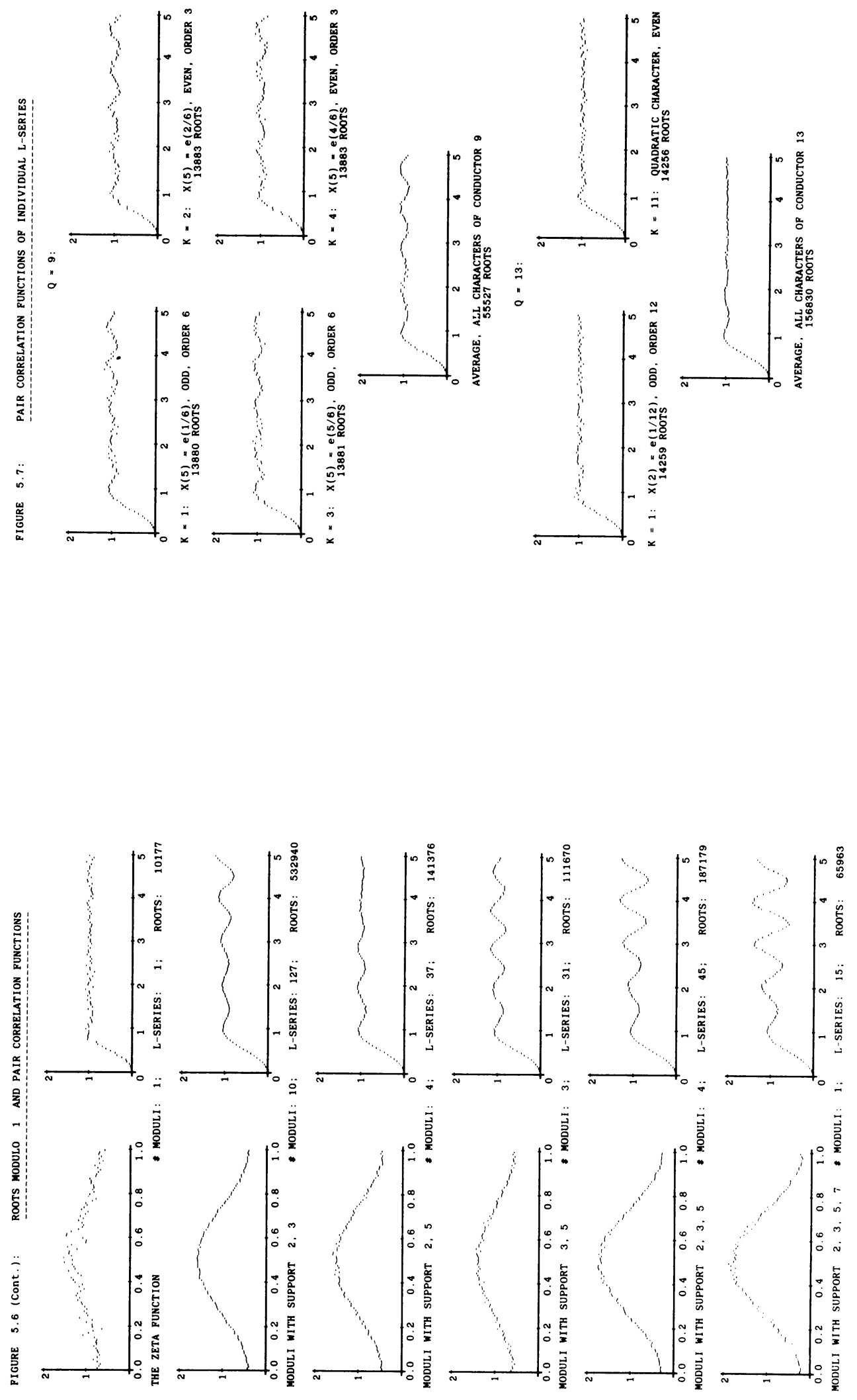

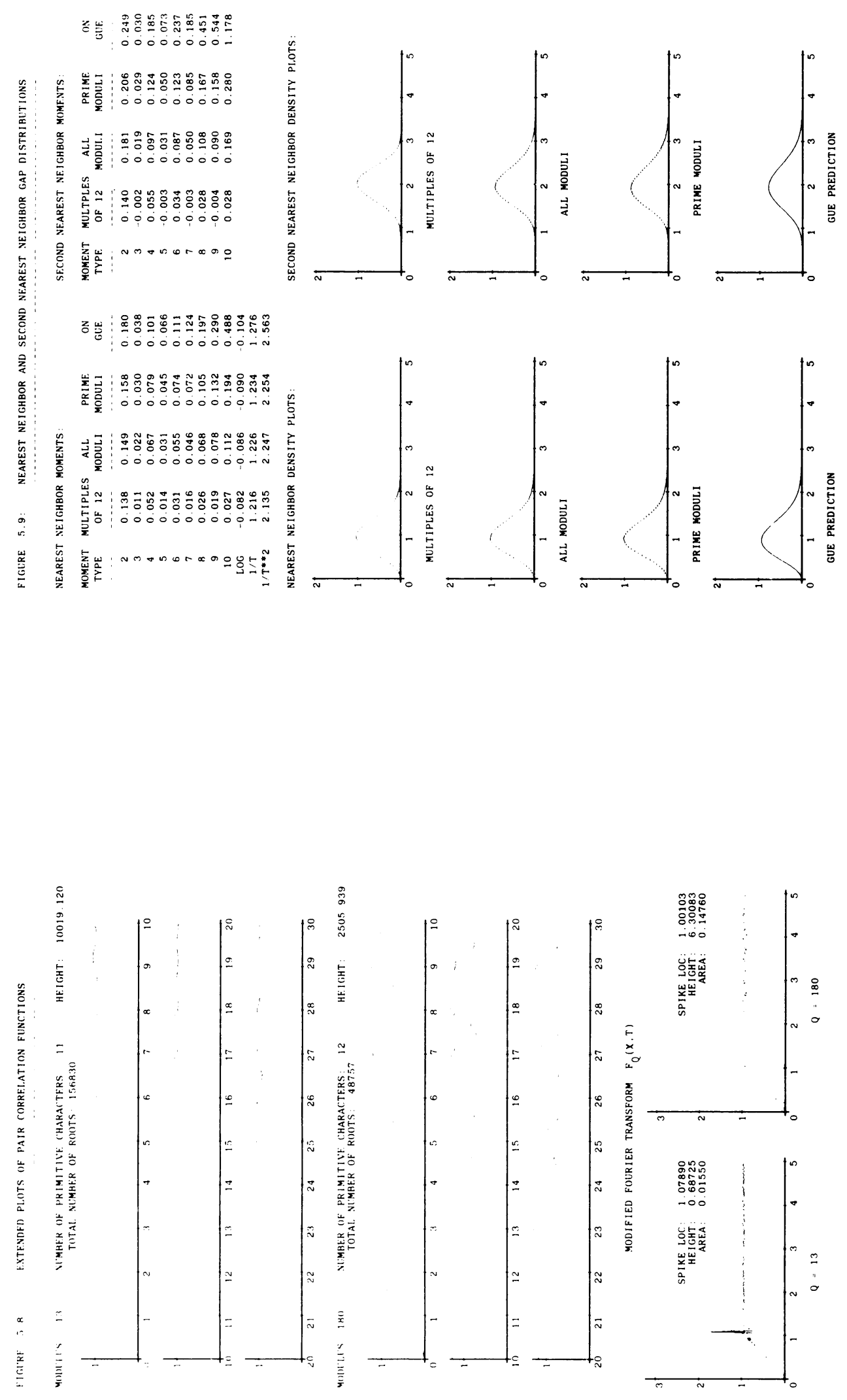

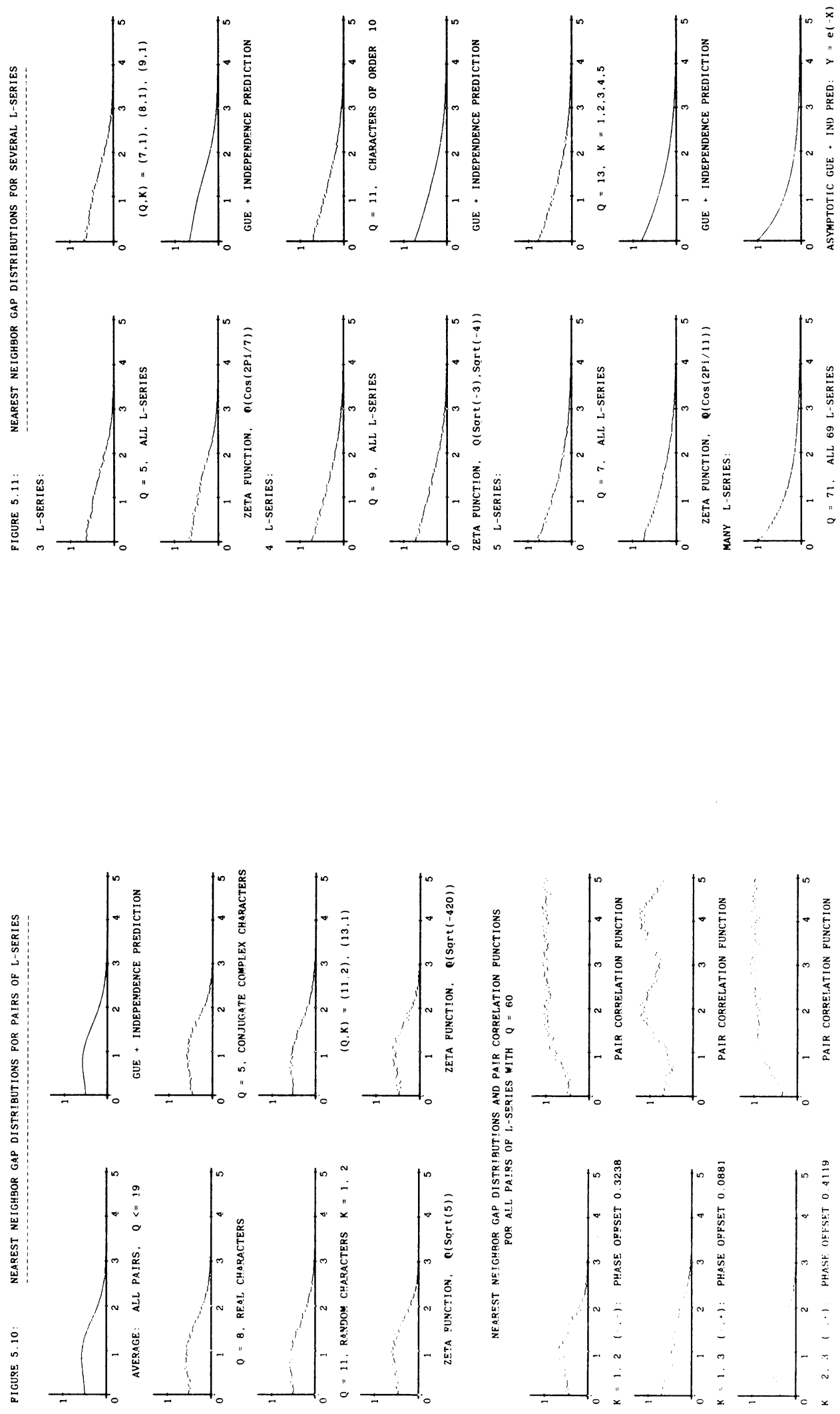